\title{
Osteotomien im Gesichtsskelett - Basis der orthognathen Chirurgie: Befunderhebung, präoperative Planung und individuelle Realisation bei Korrekturoperationen von Kiefer- und Gesichtsdeformitäten
}

\author{
Martin Roser, Carl Peter Cornelius, Gerd Gehrke, Michael Ehrenfeld, Gerson Mast
}

\section{Zusammenfassung}

Umstellungsosteotomien im Gesichtsschädel bilden die Grundlage skeletaler Korrekturen im Ober- und/oder Unterkiefer und begleitend damit des Profils und der Weichgewebe. Standardverfahren in der modernen orthognathen Chirurgie sind die bilaterale sagittale Spaltungsosteotomie (BSSO) im Unterkiefer und die Le-Fort-I-Osteotomie in Downfracture-Technik im Oberkiefer. Bei diesen Verfahren wird die komplette zahntragende Unterkieferspange bzw. der komplette zahntragende Oberkiefer mobilisiert, 3-dimensional reponiert und mit Osteosynthesen fixiert. Den skelettverlagernden Eingriffen zur Herstellung normaler Okklusionsverhältnisse und harmonischer Gesichtsproportionen gehen eine systematische Initialdiagnostik der verschiedenen Deformitäten, eine kieferorthopädische Vorbehandlung und eine Vorhersage und Simulation des avisierten OP-Resultats voraus. Die Sequenz aus konservativen und operativen Therapiephasen in der orthognathen Chirurgie bedarf immer eines interdisziplinären „TeamApproachs“, um die individuelle Pla- nung in bestmögliche Behandlungsergebnisse umzusetzen. Innovative Methoden, wie 3-dimensionale Kephalometrie, virtuelle OP-Simulation und ultraschallbasierte Osteotomietechniken („Piezochirurgie“) und Osteosyntheseverfahren vereinfachen die Abläufe zur Normalisierung der Kieferrelation und zur ästhetischen Harmonisierung des Gesichtsprofils.

Osteotomies in the Facial Skeleton Basis of Orthognathic Surgery Clinical Findings, Preoperative Planning and Surgical Treatment of Dentofacial Deformities Associated with Malocclusion

Osteotomies and subsequent positioning the bone partitions constitute the fundamental form of treatment in skeletal deformities of the mandible and the lower midface. Corrective changes of the facial soft tissue profile and contours are performed concomitantly. The bilateral sagittal split osteotomy and the Le-Fort I osteotomy with down-fracture are the standard procedures in the modern orthognathic surgery armamentarium. In the process of both methods the tooth bearing segments either consisting of the mandibular body or the complete maxillary base are mobilised, three-dimensionally repositioned and fixed with plates and screws. Skeletal shifting and repositioning to achieve normal occlusion and facial harmony must be preceded by a systematic clinical work-up and classification of the dentofacial deformity. The next steps are orthodontic preparation for surgery and the final planning including prediction and simulation of the surgical outcome. An interdisciplinary team approach (orthodontists, maxillofacial surgeons, dental and ENT specialists) is essential to guide the patient through the sequence of conservative and surgical therapy and to take into account the individual priorities and optimal results. Innovations, such as three-dimensional cephalometry, complete virtual surgery simulation, ultrasonic osteotomy techniques ("piezo-surgery") and versatile osteosynthesis procedures facilitate the protocol to normalise the jaw relations and occlusion as prerequisite for the aesthetic harmony of the facial profile.

\section{Einleitung und Begriffsbestimmung}

Die „orthognathe Chirurgie“, oder „kieferorthopädische (KFO) Chirurgie“ "bzw. „Dysgnathiechirurgie“ umfasst operative Korrekturen des Ober- und/oder Unterkiefers und des angrenzenden Schädelskeletts bei Formanomalien und Fehlstellungen mit Malokklusion (Fehlbiss), die mit einer konservativ orthodontischen bzw. kieferorthopädischen Thera-

OP-JOURNAL 2011; 27: 200-221

(c) Georg Thieme Verlag KG Stuttgart · New York DOI http://dx.doi.org/10.1055/s-0031-1280410 pie allein nicht zufriedenstellend behandelt werden können.

Die Umstellungsosteotomien sollen die Verzahnung und Kaufunktion optimieren und darüber hinaus positive Effekte auf den Zahnerhalt sowie die Ästhetik von Gesichtsproportionen und Weichgewebeprofil haben.

\section{Dysgnathieformen - Systematik und wichtige Grundformen skeletaler Anomalien}

Die Mehrzahl der dentofazialen Anomalien ist alleinige Folge von Zahnfehlstellungen und primär im Alveolarfortsatz- bereich lokalisiert. Bei Betrachtung des seitlichen Gesichtsprofils können ähnliche Situationen entstehen wie bei Dysgnathien. Die Zähne haben die Tendenz, skeletale Anomalien auf dentoalveolärem Niveau durch vertikale Positionsanpassung oder Kippung in der Sagittalund Transversalebene zu kompensieren.

Eine Differenzierung von skeletalen Anomalien bzw. Dysgnathien von primären oder kompensatorischen dentoalveolären Anomalien bei der Befunderhebung ist die entscheidende Voraussetzung für die Planung einer geeigneten Therapie (kieferorthopädisch/orthodontisch vs. 


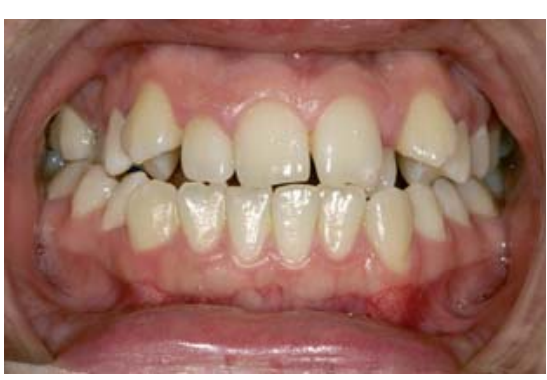

Abb. 1 Mandibuläre Prognathie, 21-jährige Patientin, Frontalansicht: Kantenbiss im Frontzahnbereich, transversaler Engstand im Oberkiefer mit Kreuzbiss im Prämolarenbereich rechts.

kombiniert orthodontisch-orthognathchirurgisch).

Einer skeletalen Dysgnathie können morphologisch und ätiologisch (kongenital oder erworben) unterschiedliche Krankheitsbilder zugrunde liegen; dabei bestehen Diskrepanzen in der Größe und Lage von Ober- und Unterkiefer zueinander und zum gesamten Gesichtsschädel [1].

Die Lageanomalien im Bereich der Kieferbasen spielen sich dabei in verschiedenen Dimensionen ab: sagittal (Distalbiss, Mesialbiss), transversal (Kreuzbiss, bukkale oder linguale Non-Okklusion), vertikal (Tiefbiss-Klausognathie, Offener-Biss-Apertognathie). Entsprechend den 6 räumlichen Freiheitsgraden sind außerdem Rotationen der Kieferbasen um die senkrecht zu diesen Ebenen stehenden Achsen möglich (vgl. Abb. 10).

Dysgnathien bzw. Gesichtsskelettanomalien gibt es in zahllosen Varianten, wenn man Größendiskrepanzen, verschiedene Lageanomalien, die Kombinationsmöglichkeiten im Ober- und Unterkiefer sowie Asymmetrien in den 3 Raumebenen und zusätzliche dentoalveoläre Abweichungen berücksichtigt.

Für einige besonders markante Grundformen werden immer noch historischtradierte Begriffe verwendet, die sich nicht durchweg an der 3-dimensionalen Architektur des Gesichtsschädels oder den Kieferrelationen orientieren, sondern an äußerlich sichtbaren Symptomen (Beispiel: Progenie oder Laterogenie für vorstehendes Kinn bzw. Seitabweichung der Kinnmitte).

Dysgnathien können sowohl im Unterals auch im Oberkiefer vorkommen; symmetrische und asymmetrische Formen werden unterschieden.
Tab. 1 Skeletale Dysgnathien im Unter- und Oberkiefer.

\section{Symmetrische Dysgnathien}

Unterkiefer

Bilaterale mandibuläre Hypoplasien („Mandibular Deficiency“):

- mandibuläre Retrognathie (Synonyma: Retromandibulie, Retrogenie):

Unterkieferrücklage bei in Bezug zur Schädelbasis normal positioniertem Oberkiefer Symptome:

Profil: „fliehendes Kinn“, anscheinend: vorstehender Oberkiefer; dental: ausgeprägte sagittale Frontzahnstufe („overjet“), evtl. zusätzliche Protrusion der Oberkieferfront oder evtl. frontale Supraokklusion („overbite“); im Seitenzahngebiet Distalokklusion

- mandibuläre Mikrognathie (Synonym: Mikromandibulie): abnorm klein dimensionierter Unterkiefer

Bilaterale mandibuläre Hyperplasien („Mandibular Excess“):

- mandibuläre Prognathie (Synonyma: Antemandibulie, Progenie):

Unterkiefervorlage bei in Bezug zur Schädelbasis normal positioniertem Oberkiefer Symptome: (vgl. Abb. 1 und 2)

Profil: vorstehendes Kinn, umgekehrte Lippenstufe; dental: umgekehrter Frontzahnüberbiss (,reverse overjet“), ggf. zirkulärer Kreuzbiss, Protrusion der Oberkieferfront, Retrusion der Unterkieferfront; im Seitenzahngebiet Mesialokklusion

- mandibuläre Makrognathie (Synonym: Makromandibulie): abnorm groß dimensionierter Unterkiefer (z. B. bei Akromegalie)

\section{Oberkiefer}

Bilaterale maxilläre Hypoplasien („Maxillary Deficiency“):

- maxilläre Retrognathie (Synonym: Retromaxillie):

Oberkieferrücklage bei in Bezug zur Schädelbasis normal positioniertem Unterkiefer Symptome:

Profil: positive Lippenstufe; dental: umgekehrter Frontzahnüberbiss;

im Seitenzahngebiet Mesialokklusion

- maxilläre Mikrognathie (Synonym: Mikromaxillie): abnorm klein dimensionierter Oberkiefer in allen 3 Raumebenen (z. B. bei Lippen-Kiefer-Gaumenspalten)

- transversale Oberkieferhypoplasie: transversal zu schmaler Oberkiefer (Abb. 3)

Bilaterale maxilläre Hyperplasien („Maxillary Excess“)

- maxilläre Prognathie (Synonym: Antemaxillie):

Oberkiefervorlage bei in Bezug zur Schädelbasis normal positioniertem Unterkiefer Symptome:

Profil: vorstehende Oberlippe; dental: große sagittale Frontzahnstufe; im Seitenzahngebiet Distalokklusion

- vertikale Oberkieferhyperplasie (Synonym: Hypermaxillie):

verlängertes mittleres Gesichtsdrittel

Symptome:

Profil: kurze Oberlippe mit Lippeninkompetenz; dental: Oberkieferfrontzähne einschließlich eines breiten Zahnfleischsaums sichtbar, besonders beim Lachen "gummy smile“

\section{Asymmetrische Dysgnathien}

Unterkiefer

- unilaterale mandibuläre Hypoplasien: können prinzipiell alle Unterkieferabschnitte betreffen, meist den Gelenkfortsatz (posttraumatisch oder syndromal)

- unilaterale mandibuläre Hyperplasien:

- hemimandibuläre Elongation: Längenüberentwicklung von Kiefergelenkfortsatz, aufsteigendem Unterkieferast und Unterkieferkorpus, führt zur Rotation nach kontralateral - hemimandibuläre Hyperplasie (vgl. Abb. 4): 3-dimensionale Vergrößerung einer Unterkieferhälfte

Oberkiefer

- unilaterale Elongation des Oberkiefers: meist Folge einseitiger mandibulärer Hypoplasien als kompensatorisches Wachstum

- unilaterale Kompression des Oberkiefers: meist Folge einseitiger mandibulärer Wachstumsstörungen mit Verkürzung des aufsteigenden Astes 




Abb. 2a bis e Mandibuläre Prognathie mit geringfügiger hemimandibulärer Elongation rechts, maxilläre Retrognathie mit vertikaler Verkürzung des Oberkiefers und transversaler Kippung der Okklusionsebene.

Die häufigsten Formen sind in der nachfolgenden Tab. 1 zusammengefasst.

Kombinationen aus den in Tab. 1 aufgeführten Dysgnathieprototypen sind häufig (vgl. Abb. 2, Fallbeispiele), sodass zur umfassenden Diagnostik dentofazialer Deformitäten grundsätzlich eine 3dimensionale Erfassung und Auswertung des Gesichtsskeletts notwendig ist.

Zur Prävalenz von Malokklusionen in Europa wird in mehreren Studien aus den letzten Jahren (Schweden, Norditalien) berichtet. Zum Vorkommen von Dysgnathien mit der Notwendigkeit zur operativen Intervention gibt es hingegen keine gesicherte Datenlage. Immer wieder werden regionale Unterschiede kolportiert, so sollen Klasse-III-Dysgnathien in Süddeutschland und Österreich häufiger anzutreffen sein als im Norden. Nach Hochrechnungen auf Basis einer USamerikanischen Gesundheitsstudie über die Jahre 1989-1994 wird geschätzt, dass bei ca. 2,7\% der Bevölkerung Malokklusionen aufgrund dentofazialer Deformitäten (2\% mandibuläre Hypoplasien und/oder vertikale maxilläre Hyperplasie; 0,3\% mandibuläre Hyperplasie und/ oder maxilläre Hyperplasie; $0,3 \%$ offener Biss; 0,1\% Asymmetrien) mit Bedarf für eine chirurgische Therapie vorliegen.

\section{Befunderhebung - Vorbehandlung - OP-Planung - Nachbehandlung}

Die Diagnostik und Behandlung skeletaler Dysgnathien beginnt in der Regel 1-2 Jahre vor Durchführung der Umstellungsosteotomien unter interdisziplinärer Beteiligung von Kieferorthopädie, Logopädie-Phoniatrie, konservierender Zahnheilkunde, zahnärztlicher Prothetik und Funktionsdiagnostik, Oralchirurgie, HNO, ggf. Psychologie/Psychiatrie und MKG-Chirurgie.

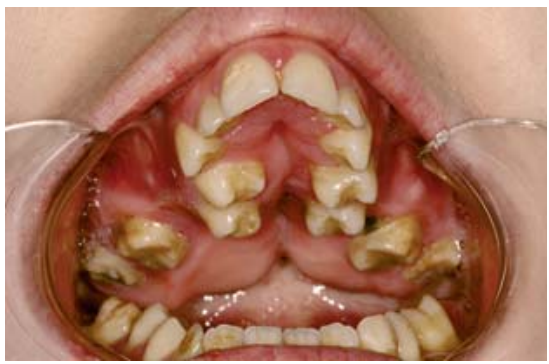

Abb. 3 Extremvariante eines transversalen Engstands im Oberkiefer (Oberkieferkompression).

Befunde - Diagnostische Unterlagen vor Behandlungsbeginn

Nach Anamnese (Fragen nach: Hauptbeschwerden, familiärem Vorkommen von dentofazialen Anomalien, Vorbehandlung, Motivation und Erwartungshaltung) und klinischer Untersuchung mit Erhebung des Gebissstatus, der Okklusionsverhältnisse bzw. der Relation von Ober- und Unterkiefer und 

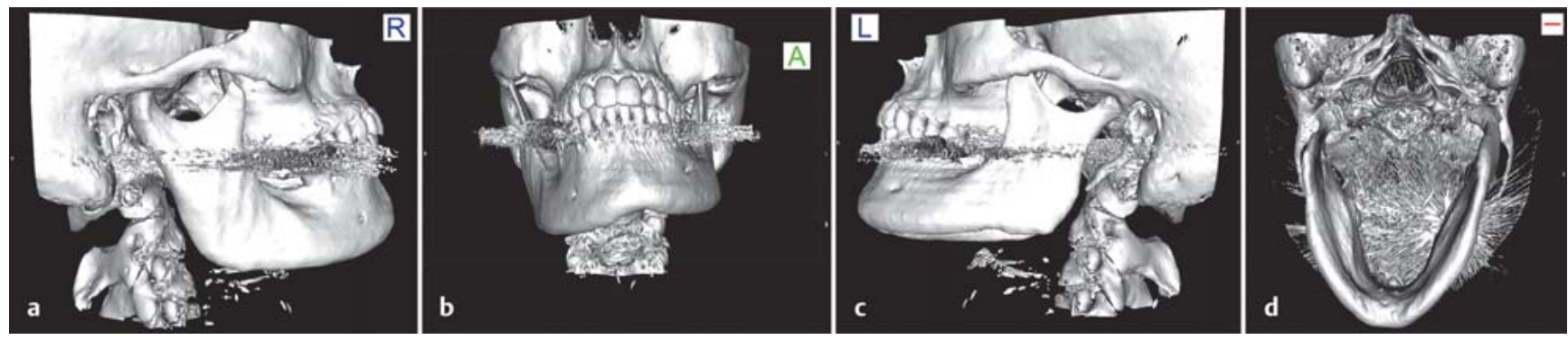

Abb. 4a bis d 3-D-CT - hemimandibuläre Hyperplasie mit Vergrößerung des Processus condylaris rechts und Vergrößerung der Unterkieferhälfte rechts in allen räumlichen Richtungen, Abweichung bzw. Rotation des Kinns nach links.
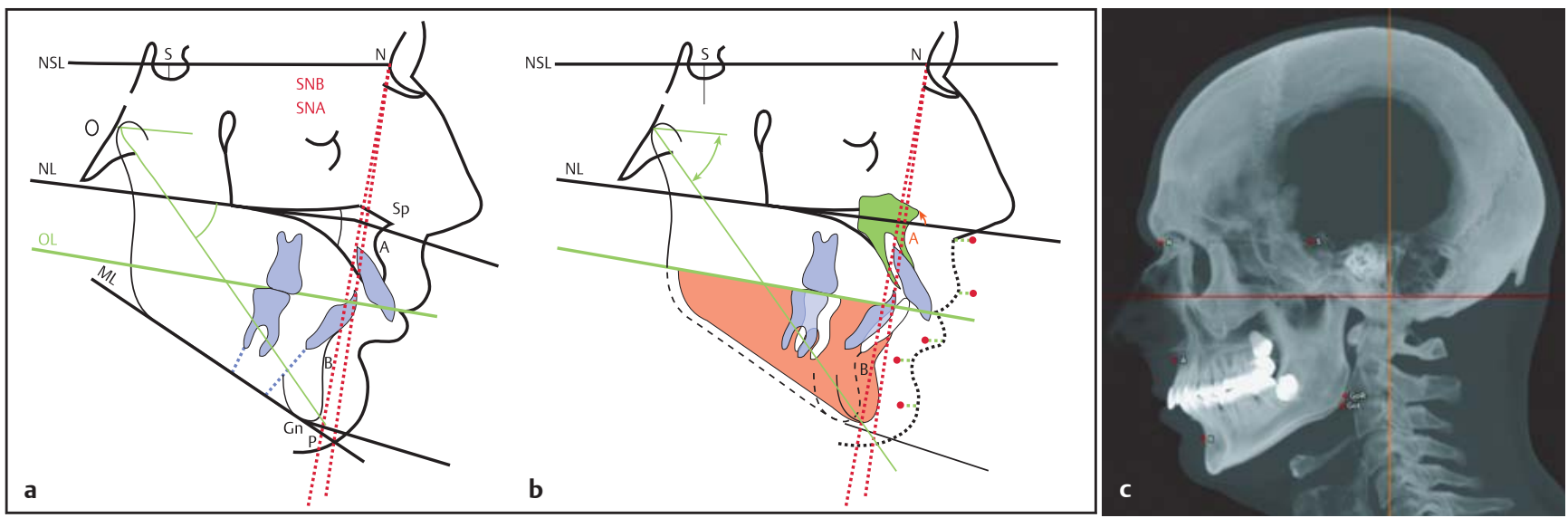

Abb. $\mathbf{5 a}$ bis c a Durchzeichnung eines Fernröntgenseitenbilds mit Eintragung von Referenzpunkten und Referenzlinien zum Beginn der Therapie (mandibuläre Retrognathie, große, sagittale Stufe, Tiefbiss) - (modifiziert und vereinfacht nach [2]) Abbildungen von konventionellen Fernröntgenseitenbildern siehe bspw. Abb. 2; Fall 1 (Abb. 19). b Durchzeichnung des Fernröntgenseitenbilds mit grafischen Korrekturen durch Verschiebung der skeletalen Elemente (Vorverlagerung des Unterkiefers und Vertikalverschiebung des Oberkiefer-Frontzahn-Segments nach kranial) als Basis zur Profilvorhersage (seitliches Lippenprofil) (modifiziert und vereinfacht nach [2]). c Aus einem CT-Datensatz reformatiertes seitliches Fernröntgenbild mit Weichteilsilhouette und räumlichem Eindruck der Skelettkonturen.

Beurteilung des Zahnhalteapparats wird eine Panoramaschicht-Röntgenaufnahme angefertigt. Von Interesse ist außerdem eine klinische und ggf. instrumentelle Funktionsanalyse mit Registrierung der Unterkieferbewegungen. Zur Dokumentation des Ist-Zustands und zu einer ersten Festlegung des Prozedere werden Abdrücke von Ober- und Unterkiefer genommen und daraus ein Anfangsmodellpaar aus Gips hergestellt.

Während die Panoramaschicht-Röntgenaufnahme dem Screening nach intraossären pathologischen Befunden (z.B. retinierte Zähne, Wurzelanomalien, Zysten etc.) dient, wird das seitliche Fernröntgenbild zur sog. „kephalometrischen Auswertung“" und Visualisierung der skeletalen Anomalie verwendet (Abb. 5).

Mit seitlichen Fernröntgenbildern werden Schädelskelett und Weichgewebe in einer standardisierten Aufnahmetechnik dargestellt. In der 2-dimensionalen kephalometrischen Auswertung werden dann skeletale und dentale Referenzpunkte in der Mediansagittalebene des
Schädels bestimmt. Die Verbindung der Punkte führt zu Referenzlinien in sagittaler und vertikaler Richtung (z.B. Schädelbasislinie), die wiederum zu Winkelund Streckenmessungen benutzt werden und quantitative Abweichungen von Normwerten feststellbar machen. Die 2-D-Kephalometrie anhand des seitlichen Fernröntgenbilds wird neuerdings immer mehr von 3-D-Auswertungsverfahren aufgrund von CT-Daten oder Kegelstrahl-Computertomografie (Cone beam CT/CBCT) bzw. digitale Volumentomografie/DVT (Abb.5c und 12) abgelöst.

Eine Kephalometrie wird zu mindestens 3 Zeitpunkten vorgenommen:

- zu Behandlungsbeginn (zur Planung der orthodontischen Vorbehandlung)

- nach Abschluss der kieferorthopädischen Vorbehandlung (zur OP-Planung mit Profilvorhersage)

- postoperativ und/oder zum Abschluss der gesamten Behandlungssequenz (zur Ergebnis-/Qualitätskontrolle)
Nach Auswertung der diagnostischen Unterlagen (Röntgenbildgebung, Fotos, Modelle) wird ein individuelles „all-inclusive"-Behandlungskonzept auf der Basis der Dysgnathieprototypen erarbeitet mit Maßgaben (Zeitbedarf, Methodik, Zielrichtung, Okklusionsposition, Koordination der Behandlungsschritte, Risikovermeidung, operative Zusatzmaßnahmen) für die orthodontisch-orthognathe Kombinationstherapie [3].

Panoramaschichtaufnahme und seitliches Fernröntgenbild sind Basisuntersuchungen zur Planung einer Umstellungsosteotomie.

\section{Vorbehandlung}

Während der kieferorthopädisch/orthodontischen Vorbehandlung werden die Zahnbögen mit festsitzenden kieferorthopädischen Multibandapparaturen ausgeformt. Um ein Maximum an skeletalen Korrekturen durchführen $\mathrm{zu}$ können, sollte eine vollständige Dekompensation der an die skeletale Anomalie angepassten Zahnfehlstellungen erreicht 
werden. Häufige Folge davon ist, dass sich die Deformität akzentuiert, worauf die Patienten vorbereitet werden müssen. Die Besonderheiten jeder Dysgnathieform müssen berücksichtigt werden. Beim frontal offenen Biss bspw. sind Extrusionen der Frontzähne zur Harmonisierung des Zahnbogens nicht immer empfehlenswert, sondern bei Entscheidung zu einer Segmentosteotomie sind stattdessen bewusst Stufen zu schaffen und die Frontzähne zu intrudieren. Vor Segmentosteotomien sollten zudem Lücken in den Interdentalräumen geöffnet werden, um das Risiko von Zahnwurzelverletzungen bei der Osteotomie zu reduzieren. In jedem Fall wichtig ist die Herstellung möglichst stabiler präoperativer okklusaler und dentaler Verhältnisse. Dem Ausgleich der dentoalveolären Fehlstellungen sind nicht selten jedoch mechanische Grenzen (z.B. durch Kaudruck) gesetzt, sodass interdisziplinär abgestimmt werden muss, welche Anomalien postoperativ, sobald eine normale Kieferrelation und Interkuspidation eingestellt ist, einfacher zu eliminieren sind.

Über eine Bissnahme (z.B. Wachsbiss zwischen den Zahnoberflächen) verschlüsselte und in einen sog. Artikulator montierte Gipsmodelle können zu Anfang der Vorbehandlung sehr hilfreich sein, um die erforderlichen Zahnbewegungen exakt festzulegen. Die Zahnreihen der Modelle werden segmentiert und die Blöcke oder Einzelzähne in einem Wachs-Setup (Abb.6) in die SollPosition gebracht.

Im Verlauf wird der aktuelle Behandlungsstand wiederholt mit Situationsmodellen überprüft, um den optimalen Operationszeitpunkt festzulegen und die definitive Operationsplanung durchzuführen.

Ebenfalls Bestandteil der Vorbehandlung sind oral-chirurgische, konservierende, implantologische und prothetische Maßnahmen. Retiniert verlagerte Weisheitszähne oder Eckzähne werden im Allgemeinen spätestens 6 Monate vor einer orthognath-chirurgischen Intervention operativ entfernt. Das Gebiss sollte kariesfrei und ggf. mit ästhetisch und funktionell perfekten Füllungen oder Kronen versorgt sein. Die mukogingivalen Verhältnisse und der Zahnhalteapparat (Parondontien) müssen saniert werden und sollten keine pathologischen Veränderungen aufweisen. Bei fehlenden Zähnen ist über eine Restau-



Abb. 6 Wax-Setup im Oberkiefer. Die notwendigen dentoalveolären und skeletalen Bewegungen werden durchgepielt. Die median sagittale Durchtrennung der Modellbasis simuliert eine chirurgische assistierte Gaumennahterweiterung zur Aufhebung eines transversalen Engstands.
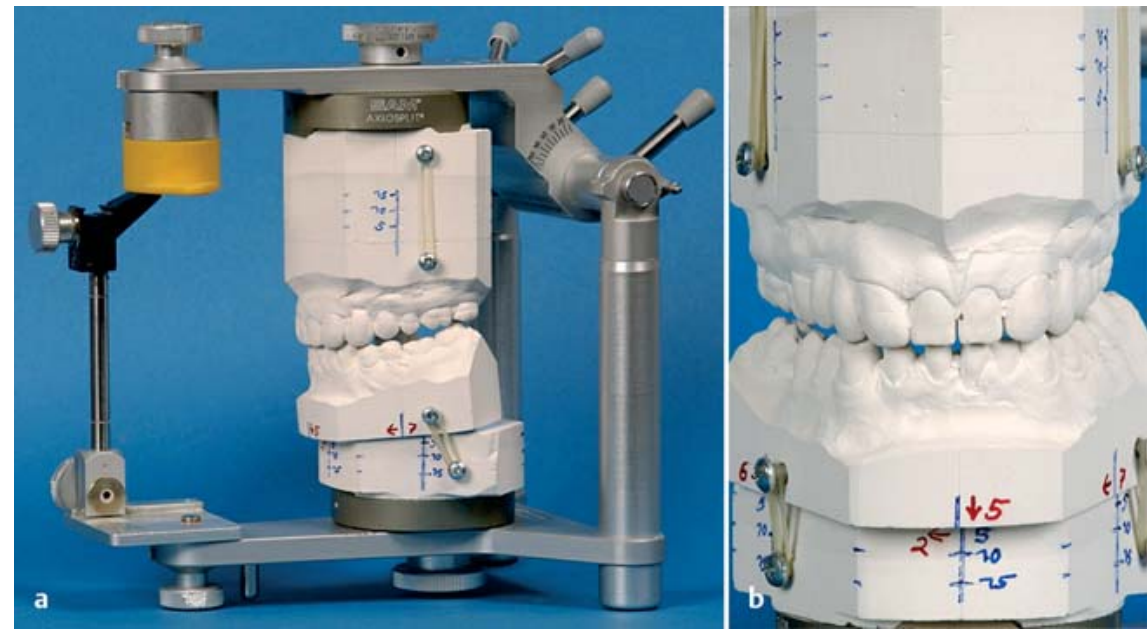

Abb. 8a und b a Modelloperaton zur monognathen Vorverlagerung des Unterkiefers. Die vertikalen Markierungslinien auf den Seitenflächen der Gipssockel erlauben es, die räumliche Bewegung des Unterkiefers zu quantifizieren (Vorverlagerung um $7 \mathrm{~mm}$ ). b Die frontale Detailansicht zeigt die Kaudalbewegung $(5 \mathrm{~mm})$ und die Schwenkung nach rechts $(2 \mathrm{~mm})$.

ration mit osseointegrierten dentalen Implantaten gegenüber Brückenprothetik oder ggf. sogar herausnehmbarem Zahnersatz zu entscheiden. Auch bei kompletter Zahnlosigkeit und Alveolarkammatrophien stellt sich gelegentlich die Indikation zu Umstellungsosteotomien. Während der Vorbehandlungsphase werden dann Totalprothesen, wenn vorhanden, mit Zahnreihen in der postoperativen Okklusionsposition versehen oder neu angefertigt. Umstellungsosteotomien im zahnlosen Kiefer lassen sich aber auch mit Knochenaugmentationen im Alveolarkammbereich zu einem präprothetisch/präimplantologischen Eingriff kombinieren.

\section{Definitive Operationsplanung}

Als frühester Zeitpunkt für einen orthognath-chirurgischen Eingriff gilt allgemein der Abschluss des Kieferwachstums. Dieser ist gleichbedeutend mit dem mittleren und höheren Teenageralter (Mädchen 16 Jahre; Jungen 18 Jahre). Verschiedentlich wird argumentiert, man könne das OP-Timing bei Hypoplasien (z. B. mandibuläre Retrognathie) ins 14. Lebensjahr vorverlegen, da das ohnehin vorhandene Wachstumsdefizit nicht weiter zunehmen würde. Bei hemimandibulären Hyperplasien oder Elongationen wird der Operationszeitpunkt jenseits des 20. Lebensjahrs gelegt, da die für diese Deformität verantwortliche Wachstumsaktivität im Kondylusbereich 


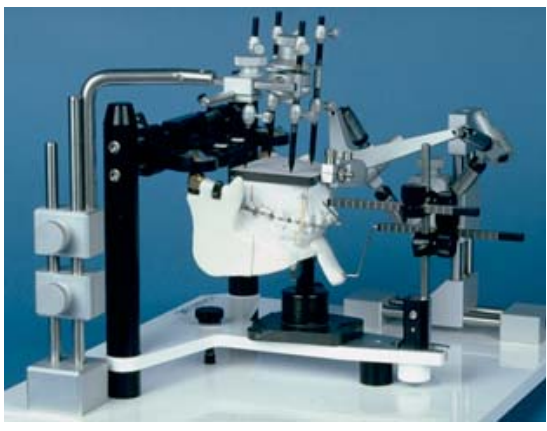

Abb. 9 3-dimensionaler Orthognathic Surgery Simulator (3D-OSS nach Krenkel/Lixl, Salzburg) [4].

erst längerfristig sistiert oder ansonsten durch ein „Shaving“ des Kiefergelenkknorpels zum Stillstand gebracht werden muss. Eine klar definierte obere Altersgrenze für orthognathe Operationen gibt es nicht.

Ist die Vorbehandlung soweit abgeschlossen, werden nach Abdrucknahme Gipsmodellpaare (Ober- und Unterkiefer) hergestellt und nach einer Gesichtsbogenübertragung in Relation zur Schädelbasis (d.h. einer Verbindungslinie zwischen äußerem Gehörgang und Infraorbitalrand) und nach einer Bissnahme zur Registrierung der Interkuspidation (Verzahnung bzw. Relation von Ober- und Unterkiefer) auf Gipssockeln in einen Artikulator (Abb. 7) montiert.

Artikulatoren sind mechanische Geräte, mit denen sich die Kiefergelenksbewegungen nachahmen lassen. Auf dem Gipssockel werden Referenzlinien angezeichnet, sodass die Verschiebungen von Ober- und/oder Unterkiefer während der Modelloperation bestimmt werden können (Abb. 8).

Die 3-dimensionale Neupositionierung kann monognath (= Umstellungsosteotomie entweder nur im Oberkiefer oder nur im Unterkiefer) oder bignath (früher: bimaxillär = Umstellungsosteotomien im Oberkiefer und im Unterkiefer) vorgenommen werden.

Für die Modelloperation vor bignathen Eingriffen sind 3-dimensionale Simulatoren immer noch interessant, weil sie eine didaktisch sehr anschauliche und kontinuierliche Einstellung der Segmente einschließlich mandibulärer Skelettanteile bieten. Beim dem hier abgebildeten 3-D-OSS [4] wird die definitive Okklusionsposition schon vor Beginn der eigentlichen OP-Simulation eingestellt und der danach resultierende Block aus

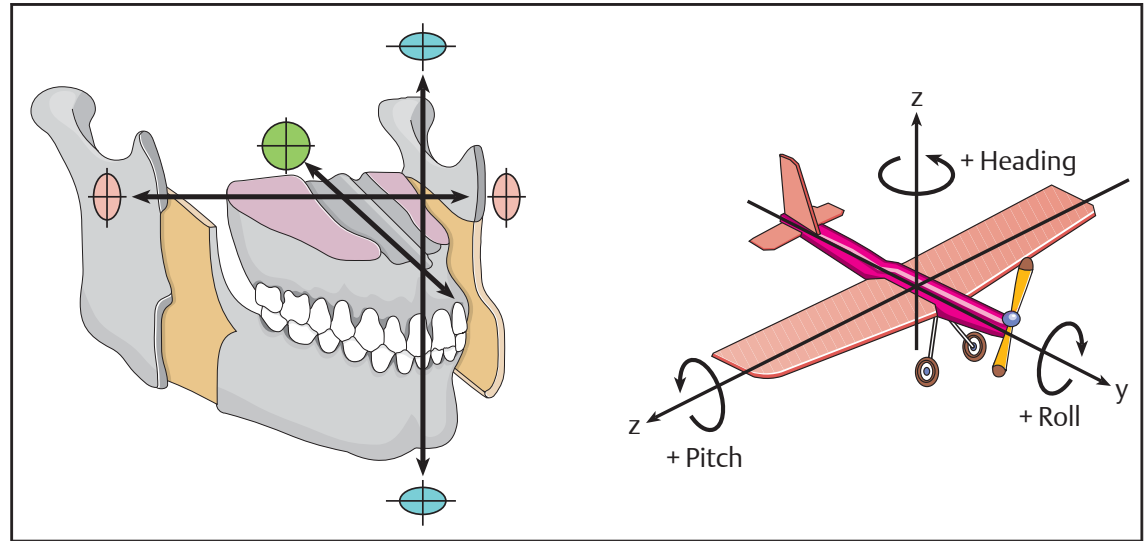

Abb.10 Mandibulomaxillärer Block im Schema. Insgesamt bestehen 6 Freiheitsgrade für die räumlichen Bewegungen.

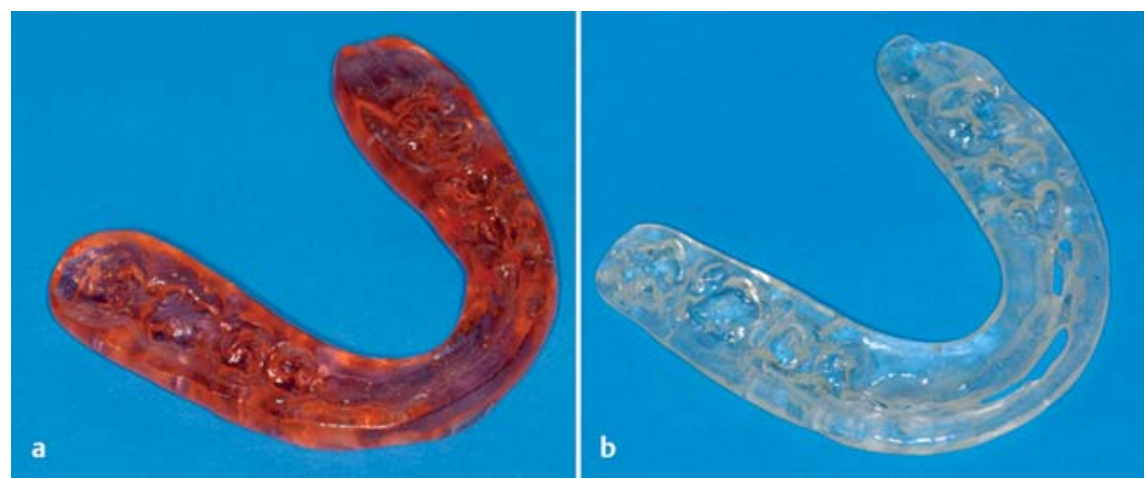

Abb. 11a und b Interokklusale Splints: Neben einem Ausgangssplint werden zur bignathen Umstellungsosteotomie 2 farbkodierte Splints verwendet („Doppelsplintmethode“). Mit dem sog. Zwischensplint (links) wird der im 1. OP-Schritt osteotomierte und mobilisierte Kiefer (i. d. R. der Oberkiefer) räumlich positioniert und über Drahtligaturen oder straffe Gummizüge am Gegenkiefer fixiert. Mit dem definitiven Splint oder sog. Zielsplint (rechts) wird der im 2. OP-Schritt osteotomierte Kiefer (i. d. R. der Unterkiefer) in die Okklusion gestellt.

Ober- und Unterkiefer („mandibulomaxillärer Komplex“) mithilfe eines 3-dimensional verstellbaren Armes in Bezug zu den aufsteigenden Unterkieferästen räumlich verschoben (Abb.9 und 10). Beide Unterkieferäste sind im Modus einer sagittalen Spaltungsosteotomie (s.u.) geteilt und die gelenktragenden Segmente mit einer Kondylus-Fossa-Verbindung aufgehängt. Die Bewegungen des mandibulomaxillären Blocks können mit einer Reihe von Skalen, Messzeigern und Winkelmessuhren präzise registriert werden. Hinter dem äußerst kompliziert anmutenden 3-D-OSS verbirgt sich der einfache Gedanke, eine Oberkieferposition zu finden, die nicht nur $\mathrm{zu}$ einem ästhetisch befriedigenden $\mathrm{Ge}-$ sichtsprofil führt, sondern eine optimale Anlagerung der Osteotomieflächen („Bony Interface“) im Bereich der aufsteigenden Unterkieferäste erlaubt, ohne dass es zu gravierenden Änderungen der Kiefergelenkposition kommt. Das lässt sich mit ästhetisch unauffälligen Ausgleichsbewegungen des mandibuloma- xillären Blocks bewerkstelligen. Bei asymmetrischen Dysgnathien erweisen sich v.a. Rotationen um eine Vertikalachse zwischen den Oberkieferschneidezähnen als sehr effektiv.

Translationsbewegungen sind entlang der $\mathrm{x}-, \mathrm{y}$ - und z-Achse möglich. Zusätzlich können Rotationsbewegungen um alle 3 Achsen durchgeführt werden (in Korrelation von Flugtechnik und orthognather Chirurgie setzen sich folgende Begriffe durch: y-Achse bzw. Drehung des Zahnbogens um die Vertikalachse = Yaw; x-Achse bzw. Drehung der Okklusalebene um die Transversalachse = Pitch, z-Achse bzw. Transversalkippung um die Sagittalachse $=$ Roll) .

Während der Modelloperation bzw. Simulation der Umstellungsosteotomien werden die in der präoperativen kephalometrischen Analyse ermittelten Bewegungen (Rotationen und Wegstrecken) des Ober- und Unterkiefers auf die montierten Gipsmodelle übertragen. Dazu 




Abb. 12a bis c a 3-D-Rekonstruktion einer Dysgnathie (mandibuläre Retrognathie) auf der Basis eines CT-Datensatzes in seitlicher Projektion. b Simulation einer Unterkiefervorverlagerung (sagittale Spaltung). c Vorhersage des Weichgewebeprofils in Korrelation zur skeletalen Verschiebung.

werden die Modelle von den Gipssockeln getrennt, die Gipssockel ggf. getrimmt und die Modelle in der Soll-Position wieder befestigt.

Zur Übertragung der Soll-Position des Ober- und/oder Unterkiefermodells auf die intraoperative Situation werden interokklusale Splints (Abb. 11) bzw. interokklusale Wafer benutzt. Das sind aus einem Kunststoffpolymerisat hergestellte Platten in Hufeisenform, auf deren Ober- und Unterseite das okklusale Relief der jeweils gegenüberliegenden Zahnreihen in Negativform (einem Einbiss vergleichbar) abgedrückt ist. Über diese vom Kunststoffsaum des Wafers gefassten Impressionen der Zahnhöcker und Gruben wird die gegenseitige Position von Ober- und Unterkiefer verschlüsselt. In zunehmender Zahl werden Dysgnathien heutzutage bignath korrigiert. Dann wird zusätzlich zu einem Ausgangssplint und dem Zielsplint ein Intermediär- oder Zwischensplint benötigt. Mithilfe des Zwischensplints wird die Position des zuerst osteotomierten Kiefers zum noch nicht osteotomierten Gegenkiefer definiert $[1,2,4,5]$.

In den letzten Jahren findet in der orthognathen Chirurgie ein „Paradigmenwechsel" statt [5-7].

Von der grafischen 2-D-Planung am lateralen Fernröntgenbild und deren geometrisch problematischer Übertragung auf 3-dimensionale Objekte während der Modelloperation geht der Weg zu einem virtuellen CAD/CAM-,,workflow“ mit konsequenter 3-D-Visualisierung in allen Phasen von Diagnostik über Prädiktion und Simulation bis zur Realisation [8-13].
Die dazu notwendigen digitalen 3-D-Datensätze werden mit bildgebenden Röntgenverfahren (CT, DVT), Photometrie, Oberflächenscanning von Kiefermodellen und intraoraler Digitalabformung der Okklusalflächen im Ober- und Unterkiefer generiert $[8,11,12]$.

Während die 3-D-Kephalometrie und die Simulation der Osteotomien und skeletalen Verlagerung mit wirklichkeitsnaher Vorhersage des Weichgewebeprofils $[8,9]$ schon vielfach etabliert ist (Abb. 12), bestehen in puncto Modelloperation und Splintherstellung häufig noch letzte „analoge Lücken“. Die „modellfreie Planung“ $[8,11]$ bedarf bisher immer noch einer aufwendigen „händischen“ Fusionierung von skeletalen Bilddaten und gescannten okklusalen Oberflächen, um ausreichend präzise interokklusale Splints virtuell zu erstellen, die sich dann mit Lasersintering oder Powder-Printing-Verfahren in ein reales Produkt umsetzen lassen [13].

Postchirurgische Kieferorthopädie Nachbehandlung

Postoperativ kann der definitive interokklusale Splint für weitere 4-6 Wochen zur Stabilisierung an der Multibandapparatur im Oberkiefer befestigt bleiben. Anschließend wird die kieferorthopädische Nachbehandlung fortgesetzt, ein Austausch der Drahtbögen ist für die Patienten dann gut tolerabel. Da die knöchernen Segmente noch geringfügig beweglich sind, dürfen vergleichsweise aggressive Maßnahmen zur definitiven Adjustierung der Okklusion erfolgen. Wegen des veränderten Knochenstoffwechsels während der ersten postopera- tiven Monate sind Zahnbewegungen leichter auszuführen. Als Faustregel gilt, dass nur etwa ein Drittel der sonst üblichen Zeitspannen notwendig sind. Kontrollen müssen daher in kürzeren Intervallen (1-2 Wochen anstelle von 4-6 Wochen) stattfinden, um ungünstige Überkorrekturen zu vermeiden.

\section{Operative Techniken in der orthognathen Chirurgie}

Das Ziel von orthognath-chirurgischen Korrekturen ist eine Normalisierung der Kieferrelation als Grundlage zur Einstellung einer Normokklusion (Regelverzahnung). Damit soll ein neuromuskuläres Gleichwicht im stomatognathen System erreicht werden und eine ästhetische Harmonisierung des Gesichtsprofils [2,3].

Orthognathe Operationen werden typischerweise über transorale operative Zugangswege durchgeführt, um äußerlich sichtbare Narben zu vermeiden.

Monognathe Osteotomien werden vorgenommen, wenn der Gegenkiefer eine 3-dimensional orthotope Position hat, d.h. „eugnath“ im Gesichtsschädel eingebaut ist.

Bei bignathen Osteotomien wird meistens mit der Oberkieferosteotomie begonnen, gefolgt von der Unterkieferosteotomie.

Standardverfahren in der modernen orthognathen Chirurgie sind die bilaterale sagittale Spaltungsosteotomie (BSSO) im Unterkiefer und die Le-Fort-I-Osteotomie in Downfracture-Technik im Oberkiefer. 


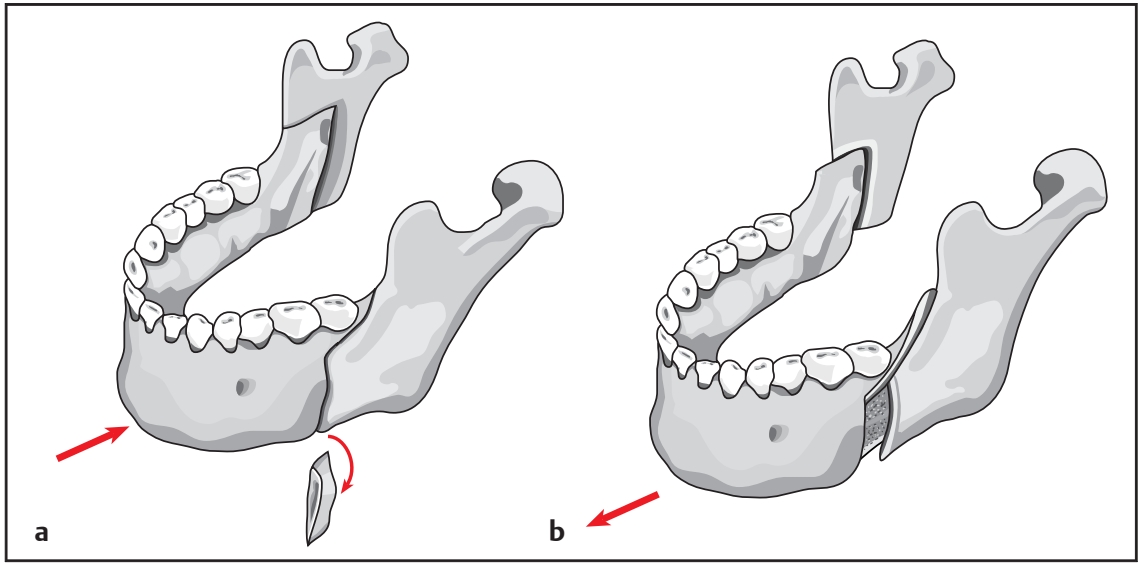

Abb. 13a und b BSSO. a Anwendung zur Unterkieferrückverlagerung mit Ostektomie einer Lamelle am bukkalen Ende der Außenkortikalis. b Anwendung zur Unterkiefervorverlagerung. Entstehung einer Knochenlücke.



Abb. 14a bis d Einige Modifikationen der sagittalen Spaltungsosteotomie im R. ascendens des Unterkiefers: a Sog. „schräge“ sagittale Spaltung nach Perthes (1924) [22], später Schuchardt (1961) [23]. b Stufenförmige sagittale Spaltung mit horizontaler Durchtrennung der Außenkortikalis in Verlängerung der Unterkiefer-Okklusionsebene nach der Idee von Obwegeser [17]. c Stufenförmige sagittale Spaltung mit vertikaler Durchtrennung der Außenkortikalis auf Höhe des letzten Molaren (Dal Pont 1961); die vertikale laterale Kortikotomie kann noch weiter vorne in der Prämolarenregion angelegt werden (Epker 1977) [21]. d Beim sog. „short split“ (Hunsuck 1968) [19] endet die Kortikotomie durch die Innenkortikalis und die sagittale Spaltung bereits hinter dem Foramen mandibulare und wird nicht bis zum Ramushinterrand geführt.

Bei diesen Verfahren wird die komplette zahntragende Unterkieferspange bzw. der komplette zahntragende Oberkiefer mobilisiert. Der Unter- und Oberkiefer können dann 3-dimensional reponiert werden, allerdings sind nicht alle Bewe- teotomien kombinieren und dienen zur Aufweitung oder Verschmälerung in transversaler Richtung oder zur vertikalen Nivellierung der Kauebene.

Bei orthodontisch nicht ausreichend beeinflussbaren transversalen Engständen im Ober- oder Unterkiefer (Schmalkiefer) wird als alleinige Maßnahme oder im Vorfeld eines orthognath-chirurgischen Eingriffs eine chirurgisch assistierte palatinale Expansion bzw. ein „Mandibular Widening“ notwendig.

In Ergänzung orthognather Operationen kommen im Einzelfall folgende Verfahren zur Anwendung: Kinnkorrekturen/Genioplastik [14], Septorhinoplastik, Nasenmuschelreduktion, Jochbeinverbreiterung, transplantate/alloplastische Augmentate zum Konturaufbau des Gesichts (Wange, Kinn, Kieferwinkel), Lippenaugmentation, Korrekturen der Submentalregion, dentale Implantate, Endoprothesen zum Kiefergelenkersatz. Eine einzeitige Durchführung stellt besondere Anforderungen an die Koordination des OP-Ablaufs (z.B. intraoperative Umintubation von nasal nach oral, Vermeidung einer Kontamination durch die Mundhöhle).

\section{Osteotomien im Unterkiefer}

Sagittale Spaltung im aufsteigenden Unterkieferast

Die beidseitige sagittale Spaltungsosteotomie (BSSO) im aufsteigenden Unterkieferast (nach Obwegeser [15-17] ist weltweit die gebräuchlichste chirurgische Technik zur Verlagerung der zahntragenden Unterkieferspange. Zur stufenförmigen sagittalen Spaltung eines $R$. ascendens werden folgende Osteotomielinien angelegt:

- Horizontaler monokortikaler Knochenschnitt durch die innere bzw. linguale Kortikalis oberhalb der Lingula zwischen Ramusvorder- und Hinterrand.

- Horizontaler, schräger oder vertikaler Knochenschnitt durch die äußere bzw. bukkale Kortikalis auf Höhe oder vor der Verbindungslinie zwischen dem inneren und äußeren Kieferwinkel.

- Vertikaler bzw. sagittal ausgerichteter Knochenschnitt entlang der Vorderkante des aufsteigenden Astes bzw. der Linea obliqua, der die Einschnitte in die linguale und bukkale Kortikalis zu einer Stufe verbindet.

Die Tiefe dieses sagittalen Einschnitts reicht bis in die Spongiosa, sodass mit 

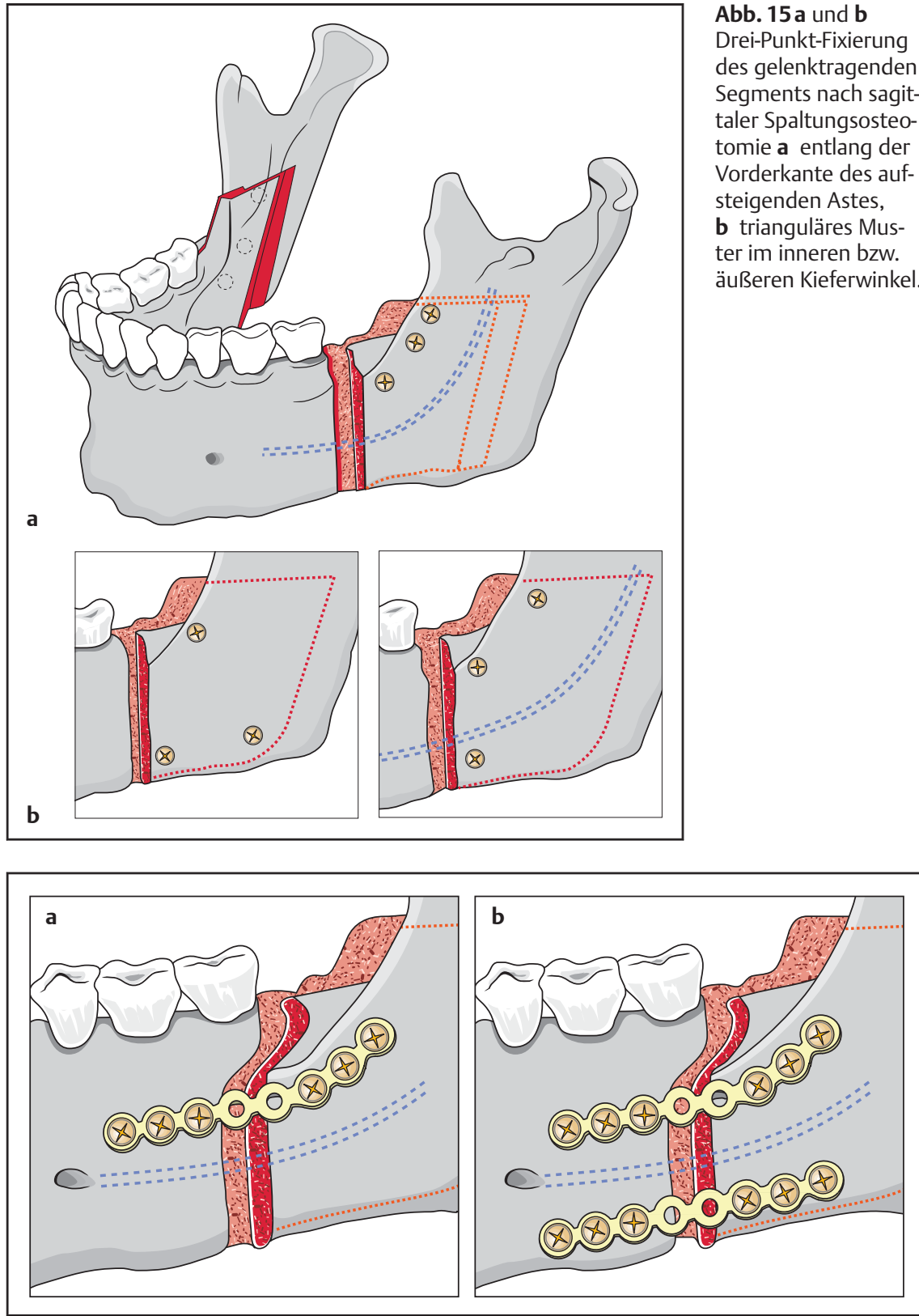

Abb. 16 a und b Miniplattenfixierung des gelenktragenden Segments nach sagittaler Spaltungsosteotomie. a Adaptationsplatte in Fortsetzung der Linea obliqua (= Oberrand). b Miniplatten in Parallelanordnung am Ober- und Basalrand.



Abb. 17 d und e Intraoperative Ansicht - Anlegen eines Bohrlochs mit einem $90^{\circ}$-Winkelbohrer zur Fixierung einer Split-Fix-Platte. Endgültig Fixation der Split-Fix-Platte.
Abb. 15 a und $b$ Drei-Punkt-Fixierung des gelenktragenden Segments nach sagittaler Spaltungsosteotomie a entlang der Vorderkante des aufsteigenden Astes,

b trianguläres Muster im inneren bzw. äußeren Kieferwinkel.

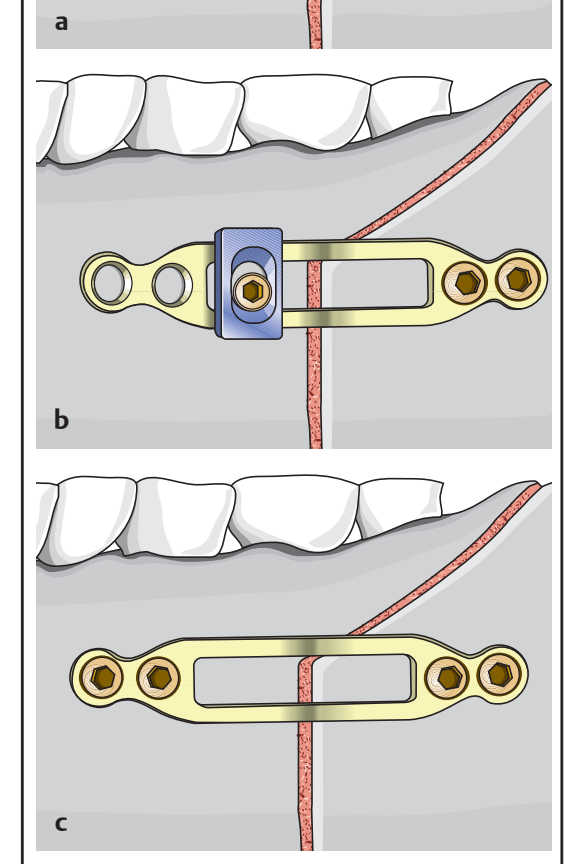

Abb. 17a bis c Split-Fix-Platte [28] im Schema. a Primär wird die Platte im Bereich der Unterkieferspange nur über ein Schiebeelement im Bereich des Doppelstegs befestigt. b Ergibt die Überprüfung eine inkorrekte Okklusion, kann der Schieber gelöst und das gelenktragende Segment in sagittaler und geringfügig auch in vertikaler Richtung neu eingestellt werden. c Sobald die Okklusion passt, werden die vorderen Plattenlöcher besetzt und der Schieber entfernt.

geeigneten Werkzeugen (herkömmlich: Osteotome oder Meißel, alternativ: Osseoskalpell oder Säge mit Spezialsägeblatt, neuerdings: Piezochirurgieansätze [18]) auf die Innenseite der bukkalen Knochenkompakta eingegangen werden kann. Der Knochen wird dann sukzessive entlang der Grenzfläche zwischen der äußeren Kompaktalamelle und der medial davon liegenden Spongiosa aufgedehnt bzw. aufgetrennt, um die sagittale Spaltung nach dorsal in Richtung auf den Ramushinterrand allmählich zu komplettieren. Durch Querspreizung lässt sich der Osteotomiespalt zunehmend besser einsehen und feststellen, ob der Mandibularkanal eröffnet wurde und ob der N. alveolaris inferior freiliegt. Der intraossäre Verlauf des Mandibularkanals ist variabel. Der Kanal liegt kei- 


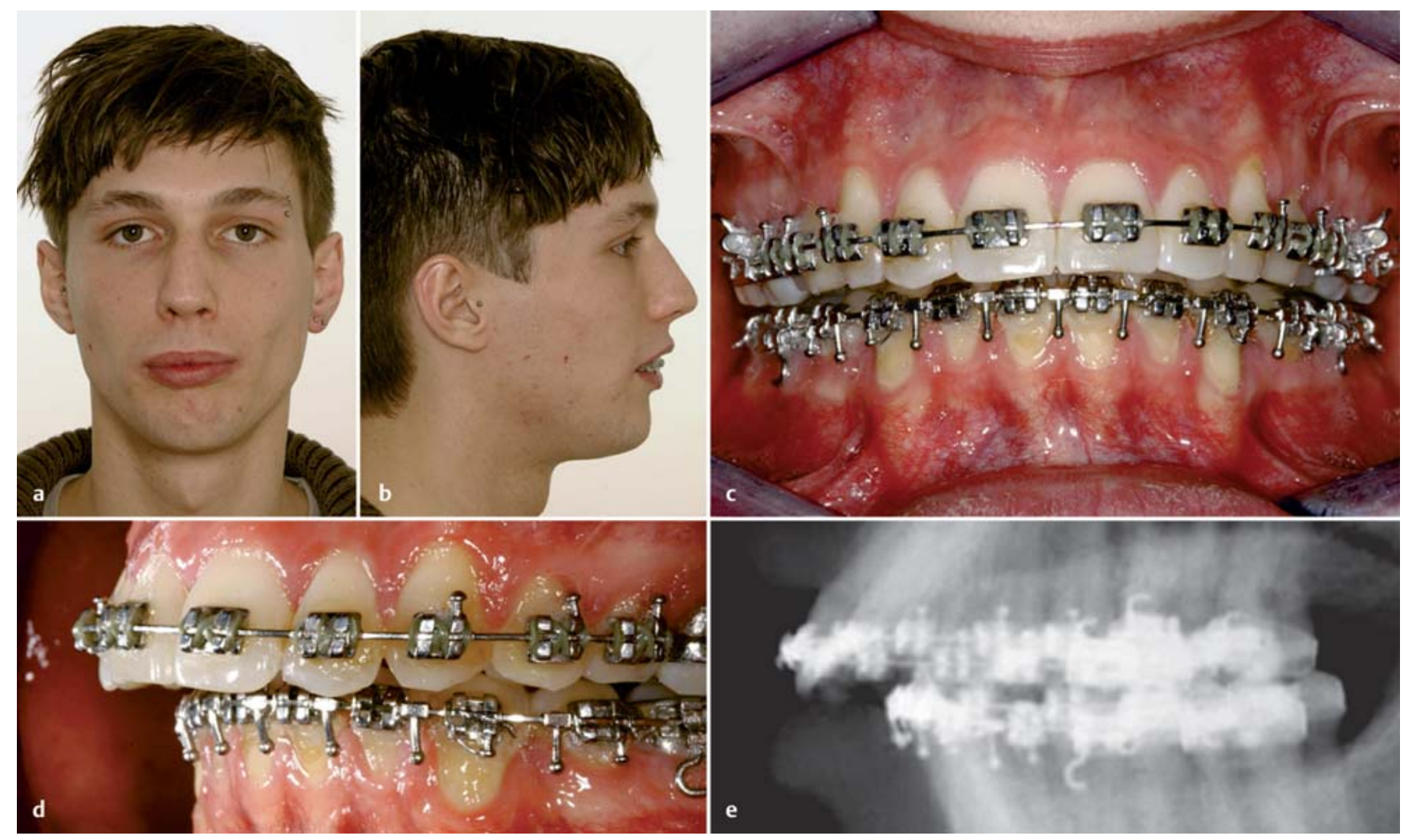

Abb. 18a bis e Präoperative Befunde Fall 1. a, b Erschwerter Mundschluss, deutlich negative Lippentreppe und ausgeprägte Supramentalfalte. c, d Okklusion mit großer Frontzahnstufe. e Fernröntgenseitenbild mit Rücklage des Unterkiefers und alveolärer Protrusion der Oberkieferfront.

neswegs immer in dem von Spongiosa bedeckten medialen Segment, sondern die bukkale Kompakta bildet nicht selten die äußere Kanalwand. Der N. alveolaris inferior wird während der Spaltung dann zwangsläufig freigelegt und muss nach medial verlagert werden, um Läsionen zu vermeiden. Schließlich wird das gelenktragende äußere Segment lateralwärts mobilisiert, um abschließend zu kontrollieren, ob die Knochenspaltung regulär verläuft oder ein „bad split“ vorliegt, woraus sich spezielle Erfordernisse im OP-Fortgang ergeben können.

Nach der sagittalen Spaltung auf beiden Seiten resultieren insgesamt 3 Unterkieferteilstücke (Abb. 13): 2 gelenktragende Ramussegmente mit je 1 Außenlamelle vorne und die Unterkieferspange mit dem Zahnbogen und 2 Innenlamellen hinten.

Die nach Obwegeser (1957) [15-17] benannte Methodik hat einige Modifikationen erfahren. Die Unterschiede liegen in einer Verkürzung des Osteotomiespalts in der Dorsalausdehnung (,short split“ Hunsuck 1968) [19] und/oder in der kaudoventralen Ausdehnung der bukkalen Knochenlamellen (Dal Pont 1961 [20], Epker 1977 [21]) und der dadurch entstehenden Veränderung der KnochenAnlagerungsflächen (Abb. 14).
Nach einer bilateralen sagittalen Spaltung der Unterkiefer-Rami kann der zahntragende Unterkieferbogen mit dem Zielsplint in die präoperativ geplante Soll-Position zum Oberkiefer gebracht und durch Drahtligaturen („mandibulomaxilläre Fixation“) daran gesichert werden.

Bei einer Rückverlagerung (Setback) der Mandibula müssen die Vorderkanten der bukkalen Lamellen an den Ramussegmenten eingekürzt werden, bei einer Vorverlagerung (Advancement) ergeben sich Diastasen an den gleichen Stellen in der Außenkortikalis.

Die Osteosynthesen erfolgen entweder transoral bspw. mithilfe eines $90^{\circ}$-Winkelschraubendrehers (vgl. Abb.17d) oder transbukkal über einen nach Anlage eines Stichkanals eingeführten Trokar und ein spezielles Instrumentarium. Zur Stabilisierung der Segmente bieten sich mehrere Fixierungsvarianten [24-28] an:

- Bikortikale Positionierungs- oder Stellschrauben in verschiedenen Anordnungsmustern - als Tandem oder in Reihe entlang der Vorderkante des aufsteigenden Astes oder triangulär im inneren und äußeren Kieferwinkel (Abb. 15).

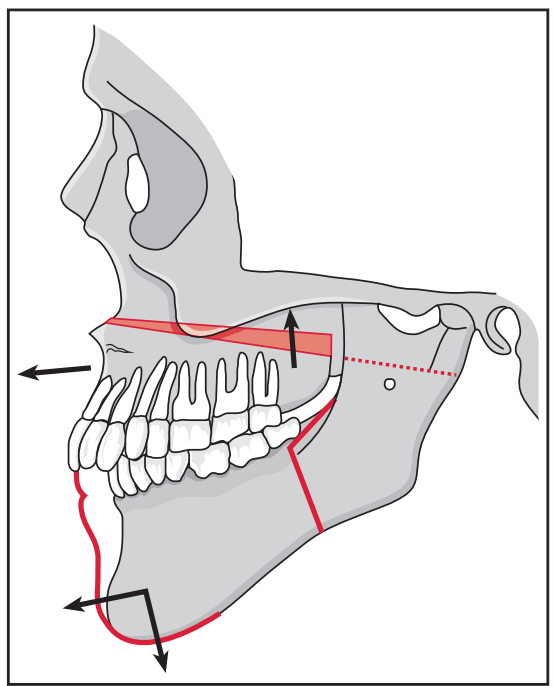

Abb. 19 Operationsschema Fall 1. Bignathe Operation - Le-Fort-I-Osteotomie zur Vorverlagerung und dorsalen Impaktierung des Oberkiefers. Dadurch Stellungskorrektur der Oberkieferfrontzähne. BSSO zur Unterkiefervorverlagerung und Erzielung einer Normokklusion.

- Monokortikal angebrachte Miniplatten entlang der Linea obliqua, ggf. auch in Parallelanordnung am Oberund Basalrand (Abb. 16).

- Adjustierbare Split-Fix-Platten, mit deren Hilfe intraoperativ die Positionierung der gelenktragenden Seg- 




Abb. 20 a bis e Postoperative Befunde Fall 1. a, b Profil frontal und lateral. c, d Okklusion. e Fernröntgenseitenbild: Osteosynthese im Unterkiefer mit einer Kombination aus Miniplatten und Positionierungsschrauben.

mente in die Gelenkgrube verändert werden kann (Abb. 17).

Die Auswahl der Fixationsmethode richtet sich im Wesentlichen nach der Ausdehnung der Osteotomie und Kontakten bzw. Lücken im Osteotomiespalt. Außer Titan kommen auch resorbierbare Materialien zur Osteosynthese infrage.

Nach Fixierung der Segmente werden die Drahtligaturen zum Oberkiefer gelöst. Die korrekte Kondylenposition sowie eine zwanglos erreichbare maximale Interkuspidation durch Bewegung des Unterkiefers wird überprüft, bevor der Wundverschluss durchgeführt wird.

Eine postoperative Ruhigstellung des Unterkiefers ist nach den genannten Osteosynthesetechniken nicht notwendig.

Die Anwendung moderner Osteosyntheseverfahren (Miniplatten, bikortikale Stellschrauben etc.) nach Unterkieferosteotomien vermeidet eine postoperative Ruhigstellung mittels intermaxillärer Fixation.

Fall 1: Patient, 19 Jahre, mandibuläre Retrognathie, maxilläre alveoläre Protrusion (Abb. 18-20)
Fall 2: Patientin, 18 Jahre, mandibuläre Lateroprognathie (asymmetrische Dysgnathie), maxilläre Retrognathie mit transversaler Hypoplasie, frontal skeletal offener Biss (Abb. 21-23)

\section{Osteotomien und Ostektomien im Unterkieferkörper}

Segment- oder Blockosteotomien und Ostektomien im Alveolarfortsatzbereich des Unterkiefers sind in zahlreichen Varianten beschrieben worden. Sie stammen aus einer Zeit, in der kieferorthopädisch-orthodontische Korrekturen nur sehr eingeschränkt möglich waren und wurden vorwiegend zum Ausgleich dentoalveolärer Anomalien eingesetzt. Heute sind diese Methoden weitgehend von orthodontisch-orthognathen Kombinationsverfahren verdrängt.

Seit Kurzem haben monokortikale Segmentierungen der Alveolarfortsätze allerdings eine Renaissance. Um orthodontische Bewegungen bei Erwachsenen zu beschleunigen, werden piezochirurgisch interdentale und subapikale Kortikotomielinien auf den Lateralflächen angelegt. Diese Technik zur Effizienzsteigerung in der kieferorthopädischen Behandlung entwickelt sich unter der Bezeichnung „speedy surgical orthodon- tics" und gilt ebenfalls als Paradigmenwechsel [5-7].

Exemplarisch erwähnt sei die frontale Alveolarfortsatz-Vorverlagerung nach Hofer (1942) [29]. Nach Extraktion der beiden vorderen Prämolaren wurde zur Bildung eines Blocks das Front-/Eckzahnsegment unter Intaktbelassung der Unterkieferbasis kastenförmig osteotomiert (Abb. 24). Bei der Vorverlagerung vergrößerten sich die Knochenlücken noch weiter und mussten mit autologen Knochenspänen gefüllt und stabilisiert werden. Von Köle wurde das gleiche Osteotomieverfahren zur Rückverlagerung des Frontzahnsegments verwendet. Nach Ostektomie der Alveole ließ sich die Zahnlücke schließen.

Zur Verkürzung des Unterkieferkörpers wurden in vorhandenen Zahnlücken oder nach der Extraktion einzelner Zähne beidseitige segmentale Ostektomien vorgenommen. Bei geradliniger Ostektomie (Dingman 1944) [30] wurde durch ein Knochenfenster der N. alveolaris inferior von lateral freigelegt und dann die Ostektomie nach lingual vervollständigt (Abb. 25).

In Weiterentwicklung der linearen Technik wurde die Segmentresektion im Un- 




Abb. 21 a bis e Präoperative Befunde Fall 2. a-c Gerades, flaches Mittelgesicht, transversaler Engstand der Oberkieferzähne, leichte Kinnabweichung nach rechts, positive Lippentreppe. d Okklusion mit frontaler Bissöffnung und Kreuzbisssituation beidseits. Zusätzlich Abweichung der Zahnmitten. e Orthopantomogramm nach Gaumennahterweiterung zur Verbreiterung des Oberkiefers.

terkieferkörper in Treppenform mit der unteren Stufe vor dem Austrittspunkt N. alveolaris inferior am Foramen mentale vorgenommen, um das Risiko einer Läsion bei der Nervenfreilegung zu umgehen [31] (Abb. 26).

\section{Osteotomien im Oberkiefer}

\section{Le-Fort-I-Osteotomie}

Das meist verwendete Operationsverfahren zur Umstellung des Oberkiefers ist die Osteotomie auf der Le-Fort-I-Ebene mit Downfracture. Beim Standardvorgehen wird der Oberkiefer in etwa nach dem Muster der von Le Fort angegebenen Bruchlinien auf Höhe der unteren Nasenapertur horizontal vom Mittelgesicht abgetrennt (Abb. 27).

Zur Downfracture wird das Oberkiefersegment vorne heruntergedrückt und aufgeklappt. Nach Mobilisation kann das Oberkiefersegment dann schubladenartig nach vorne bewegt werden. Ver-

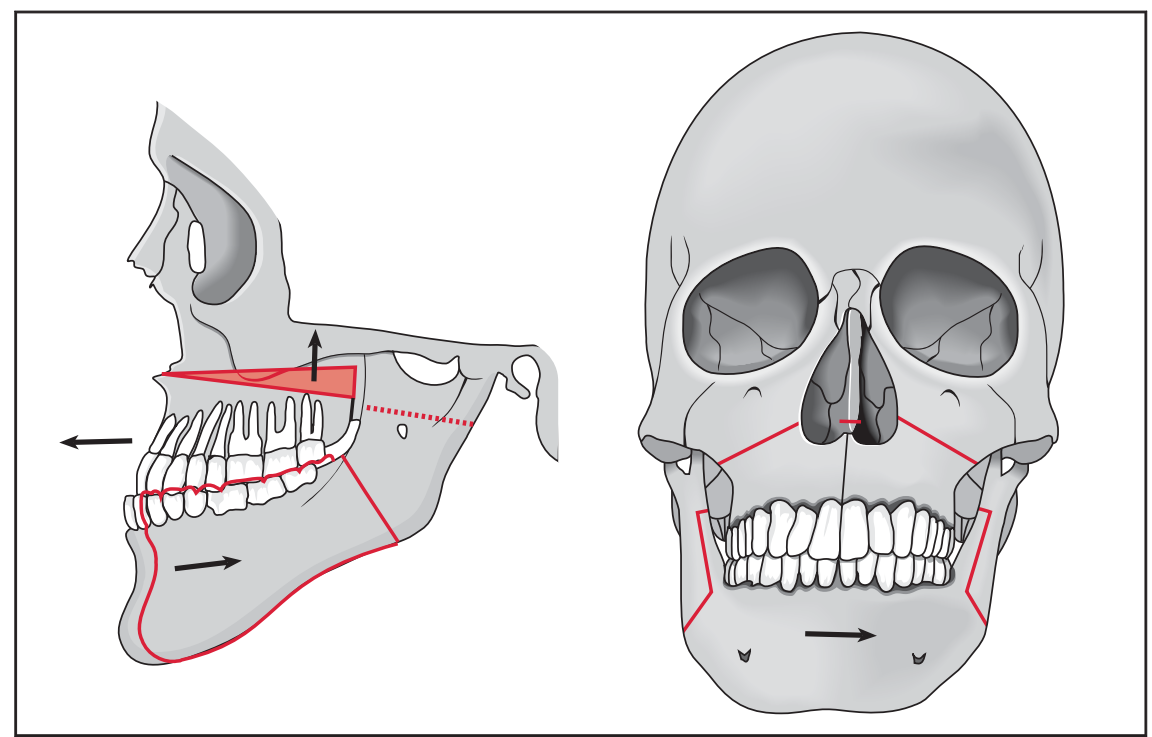

Abb. 22 Operationsschema Fall 2. Le-Fort-I-Osteotomie zur Oberkiefervorverlagerung mit dorsaler Impaktierung. BSSO zur Unterkieferrückverlagerung und Derotation nach links. 


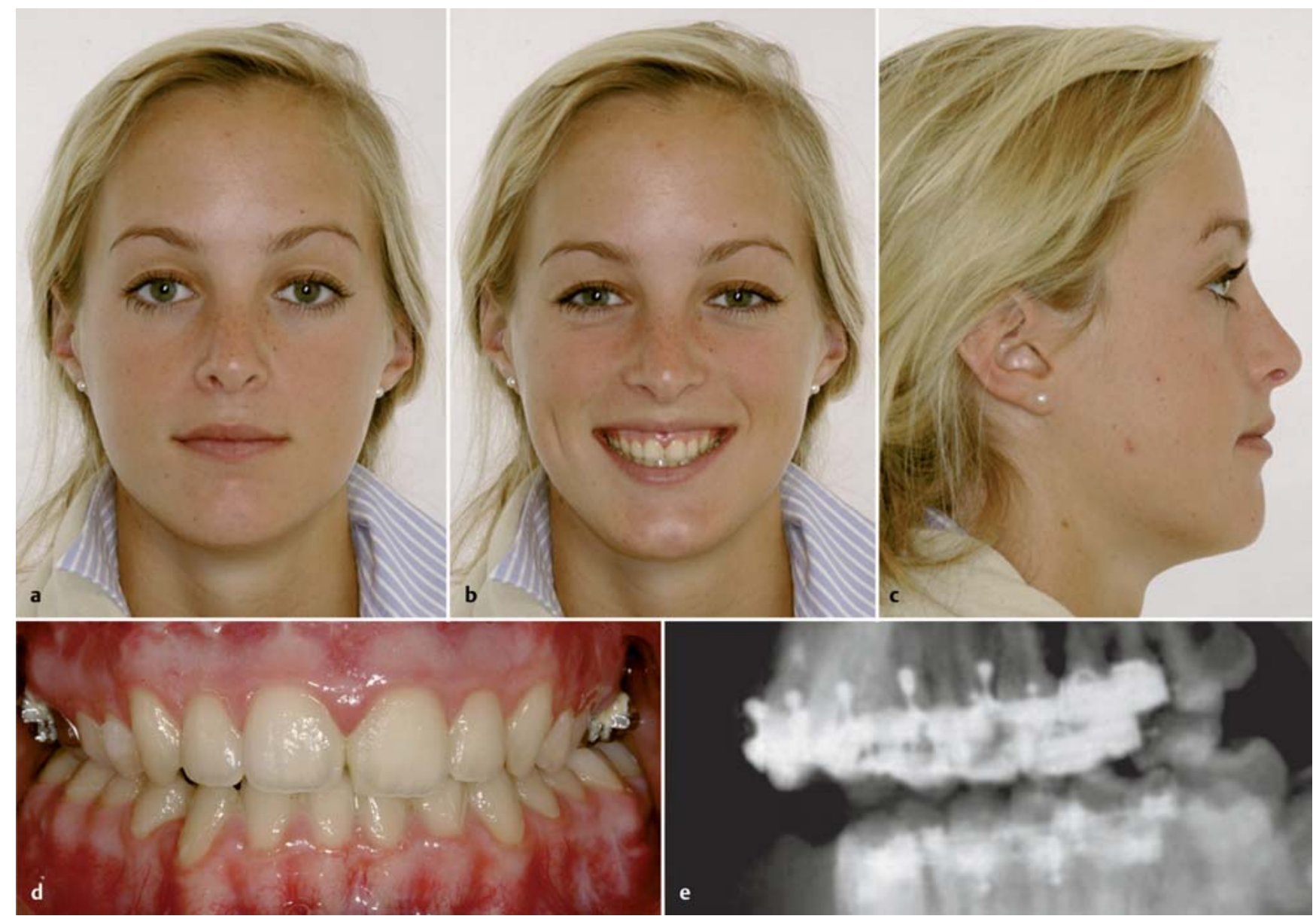

Abb. 23a bis e Postoperative Befunde Fall 2. a-c Korrigierte Gesichtsachse und Harmonisierung des Profils. d Okklusion. e Fernröntgenseitenbild Osteosynthese am Ramusvorderrand mit jeweils 3 Positionierungsschrauben.

lagerungen nach oben, nach hinten bzw. unten werden durch Ostektomien bzw. mit einer Knochentransplantation ermöglicht. Zusätzlich können Rotationsbewegungen um alle drei Achsrichtungen durchgeführt werden. Die totale Osteotomie des Oberkiefers geht auf Arbeiten von Cheever (1870) [32], Wassmund (1927, 1935) [33] und Axhausen (1934) [34] zurück. Die experimentellen Grundlagen für die Downfracture-Technik, d.h. der Nachweis einer ausreichenden Vaskularisation des Oberkiefers über die palatinale verbleibende Schleimhautbrücke, wurden erst viel später von Bell (1975) [35] erarbeitet und haben die orthognathe Chirurgie seit den 1980erJahren (Epker 1984) [36] entscheidend beeinflusst.

Die knöcherne Oberkieferbasis auf der Le-Fort-I-Ebene bzw. die fazialen Kieferhöhlenwände und die untere Zirkumferenz der knöchernen Nasenapertur werden enoral über eine umlaufende horizontale Schleimhautinzision im Vestibulum freigelegt. Die subperiostale Präparation geht bis in die Infraorbital-

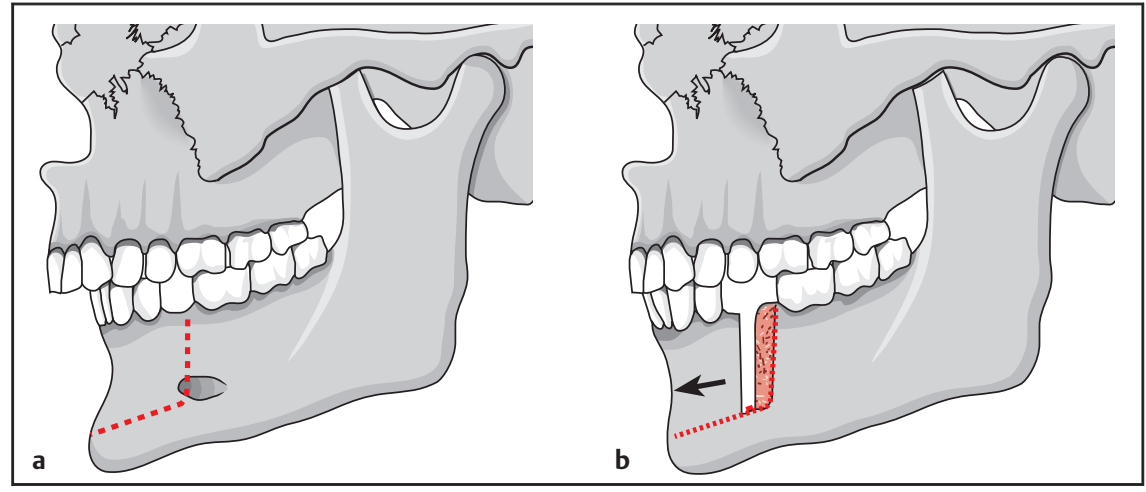

Abb. 24a und b Frontale Blockosteotomie im Unterkiefer zur Alveolarfortsatzvorverlagerung nach Hofer [29].

region sowie über die Cristae zygomaticoalveolares nach retrotubär und in die Fossae pterygopalatinae. Der Nasenschlauch wird vom Nasenboden und der seitlichen Nasenwand und vom knöchernen Septumtisch und der unteren Nasenscheidewand zur Darstellung des kaudalen Septumrands tunnelierend ausgelöst. Dann wird das knorpelige Nasenseptum bzw. Vomer vom Septumtisch bzw. vom Nasenboden separiert.
Das Design der Osteotomielinien (Höhenniveau und Neigung) durch die fazialen Kieferhöhlenwände und die Pfeilerstrukturen kann nach Richtung der beabsichtigten Neupositionierung des Le-Fort-I-Oberkiefersegments variiert werden (Abb. 28). Die Knochenschnitte durch die Maxillae werden lateral am Jochbeinansatz begonnen und nach Eröffnung der Kieferhöhle durch die faziale Kieferhöhlenwand medialwärts ge- 


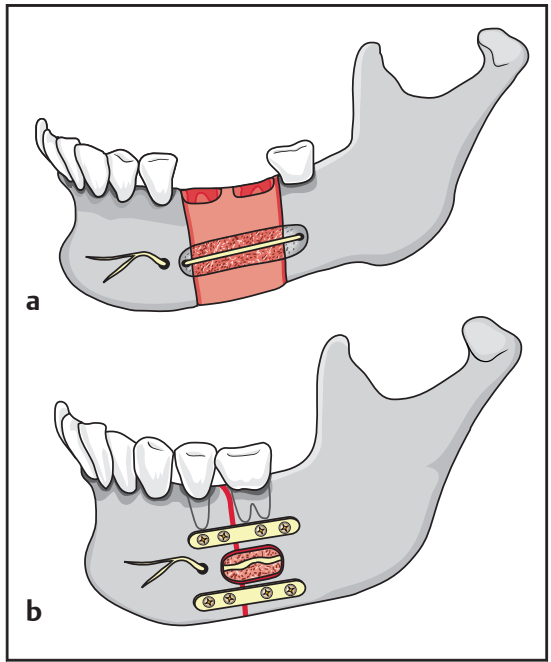

Abb. 25a und b Geradlinige Ostektomie zur Verkürzung des Unterkieferkörpers [30]. a Die linearen Osteotomielinien zur Entfernung des Segments sind rot markiert. In der Außenkortikalis ist ein Knochenfenster zur Freilegung des $\mathrm{N}$. alveolaris inferior ausgefräst. b Verkürzung des Unterkieferköpers durch Lückenschluss unter Erhalt des N. alveolaris inferior. Stabilisierung der Segmente in moderner Variante mit Miniplattenosteosynthese. Zu Zeiten der Erstbeschreibung der Methode wurden zur Stabilisierung dentale Schienenverbände und eine Ruhigstellung des Unterkiefers durch Drahtfixierung am Oberkiefer über die Dauer mehrerer Monate verwendet.

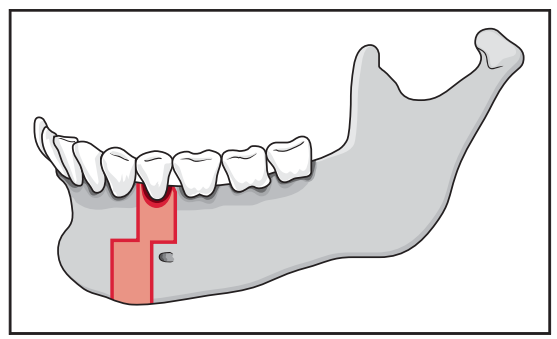

Abb. 26 Treppenförmige Ostektomie zur Verkürzung des Unterkieferkörpers. Die Ostektomiezone ist rot markiert. Die untere Stufe liegt vor dem Foramen mentale.

führt. Neben oszillierenden Sägen werden dazu Osseoskalpelle oder auch die Piezochirurgie, eine ultraschallgesteuerte Technologie, die weichgewebeschonend arbeitet und damit das Verletzungsrisiko von Nachbarstrukturen reduziert, verwendet.

Durch die „pterygomaxilläre Sprengung bzw. Separation“, in traditioneller Version blind mit einem gebogenen Spezialmeißel ausgeführt (Abb. 29) - in moderner Variante unter Sicht nach Ostektomie in der lateralen Tuberregion mit Piezochirurgieansatz -, wird das Oberkiefersegment retrotubär abgesetzt.

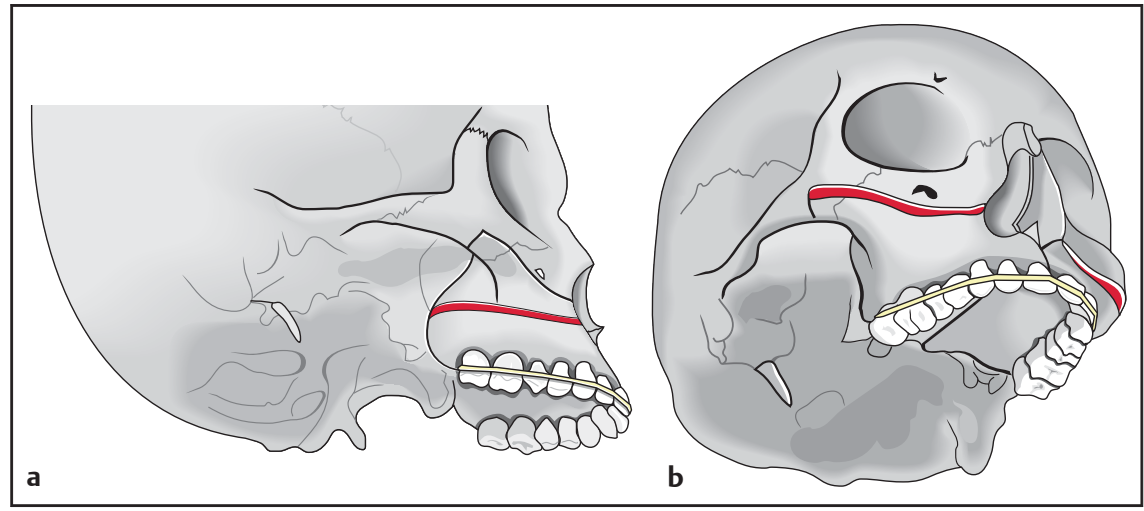

Abb. 27a und b Oberkieferosteotomie auf Le-Fort-I-Ebene. Varianten der Osteotomielinien a entsprechend eines Le-Fort-I-Frakturmusters, $\mathbf{b}$ mit Ausdehnung in den Bereich des Jochbeinkörpers beidseits.

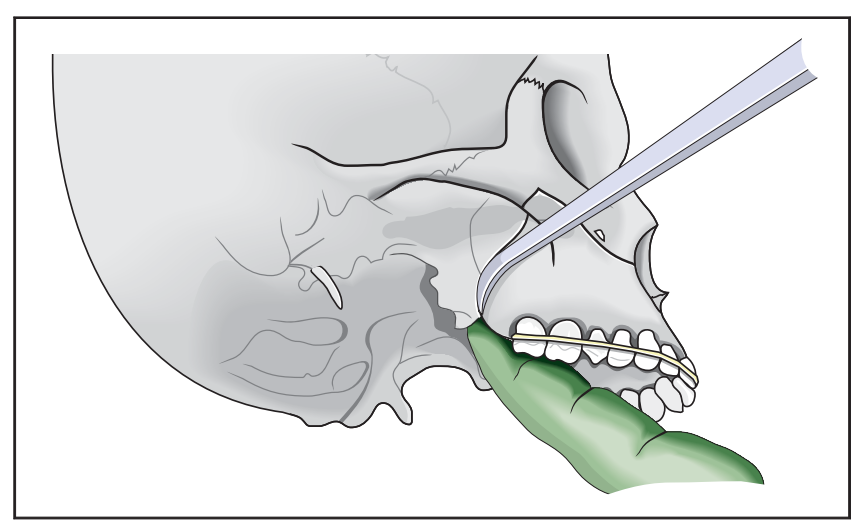

Abb. 28 Vervollständigung der Osteotomien durch „Pterygomaxilläre Sprengung" mit einem gebogenen Meißel.

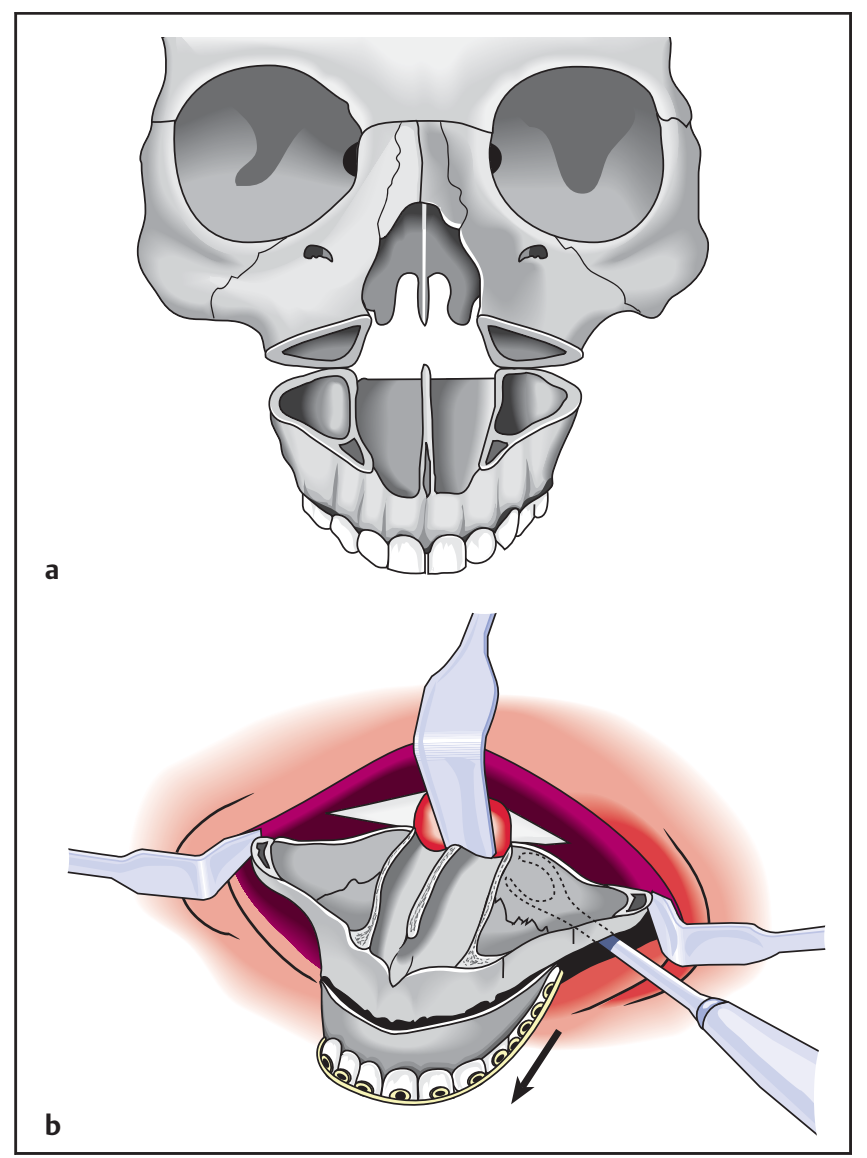

Abb. 29a und b Downfracture. a Schema: Aufklappen des Oberkiefersegments vorne. b Intraoperative Ansicht des aufgeklappten Oberkiefersegments. Einblick von oben auf Nasenboden und die Böden der beiden Kieferhöhlen. 


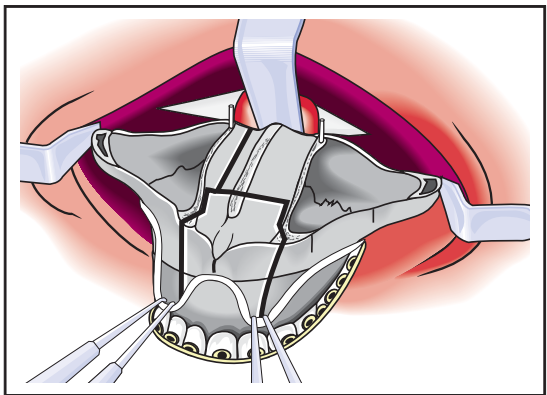

Abb. 30 Teilung des Oberkiefersegments (Three Piece Le Fort I) in Downfracture-Position.

Nun wird die Downfracture (Abb.30a) mit beiden Händen durch Fingerdruck (Kaudo-Dorsal-Richtung) auf der Vorderfläche des Oberkiefersegments und/oder durch Zug mit einem in die Nasenapertur eingesetzten Jochbeinrepositionshaken herbeigeführt. Während der Downfracture werden die letzten nicht vollständig durchtrennten Knochenlamellen im dorsal-medialen Bereich um das sphenopalatinale Gefäßbündel (A. palatina descendens) aufgebrochen, um die Osteotomie auf der Le-Fort-I-Ebene zu komplettieren.

Das Oberkiefersegment wird durch Hebel-, Dreh-, Dehn- und Vorwärtsbewegungen mit retromaxillär eingesetzten Instrumenten (Meißel, Haken) dann so weit aus seiner fissuralen Verankerung gelöst, bis es nur noch an den dorsalen Weichgeweben gestielt und zwanglos beweglich ist.

Durch das Aufklappen werden der Nasenboden und die Kieferhöhlenböden bis in den Bereich der beiden Tubera maxilla sowie die Vorderkanten der Processus pterygoidei von der Kranialseite zugänglich.

Das Le-Fort-I-Oberkiefersegment kann in der Downfracture-Position von oben in 2 oder mehrere „Stücke“ unterteilt werden (Abb. 30)

Damit werden außer einer skeletalen Lageveränderung auch dentoalveoläre Korrekturen des Oberkiefers zur Nivellierung des Zahnbogens möglich. Infrage kommen paramediane Gaumendachosteotomien beidseits, die in einen interdentalen Schnitt zwischen den mittleren Inzisivi auslaufen (sog. „2 Piece Maxilla“) oder mit einem transversalen Front-/Eckzahnsegment kombiniert werden können (sog. „3 Piece Maxilla“). Über diese Osteotomien lassen sich v.a. posterior transversale Verbreiterungen bewirken,



Abb. 31 „Autorotation“. Der okklusal eingestellte Oberkiefer wird durch Drahtligaturen mit dem Unterkiefer zum mandibulomaxillären Block vereinigt. Mit dieser Einheit werden dann symmetrische Drehbewegungen um die Kiefergelenkachse ausgeführt.

bei der 3-Piece-Osteotomie sind außerdem unterschiedliche Vertikalbewegungen und Rotationen der Segmente ausführbar.

Nach Einsetzen des intraoperativen Zielsplints (bei monomaxillärer Osteotomie) bzw. des Zwischensplints (bei bignather Osteotomie) und intermaxillärer Ruhigstellung über Drahtligaturen wird der mandibulomaxilläre Block mithilfe des sog. „Autorotations“-Manövers (vgl. Abb. 31) in die geplante Position gebracht, bevor das Oberkiefersegment kranial am Mittelgesichtsskelett fixiert wird (Abb. 32).

Die Neupositionierung in sagittaler Richtung über den Splint ist zwar unproblematisch, der vertikale und transversale Einbau wird aber von der manuell geführten Rotationsbewegung des mandibulomaxillären Blocks um eine arbiträr festgelegte Kiefergelenkachse bestimmt, wobei sich unerwünschte Abweichungen bei der Einstellung der Kondylen in die Gelenkgruben ergeben können.

Die endgültige Neupositionierung des Oberkiefers erfolgt durch die sog. Autorotation des Unterkiefers. Bei einer Kranialverlagerung kommt es gleichzeitig immer zu einem Ventralshift des Oberkiefersegments. Erfolgt die Autorotation mit einer Gelenkwalzenstellung (auch asymmetrisch) außerhalb der Fossae articulares, können gravierende Positionierungsfehler des Oberkiefers auftreten.

Üblicherweise wird das Oberkiefersegment mit insgesamt 4 L-fömigen Osteo-

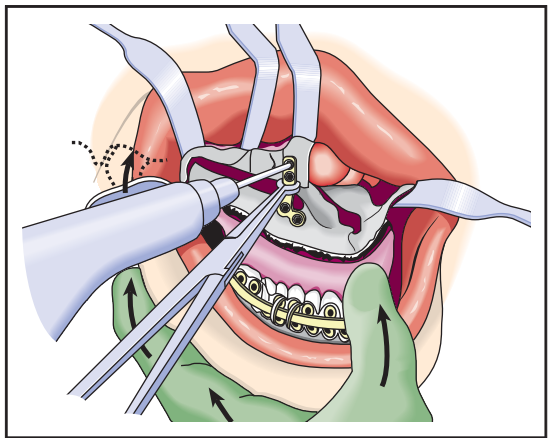

Abb. 32 Interokklusaler Splint und Drahtligaturen nach Vor- und Abwärtsverlagerung des Le-Fort-I-Segments. Beginn der Fixierung mit Miniplatten am parapiriformen Pfeiler.

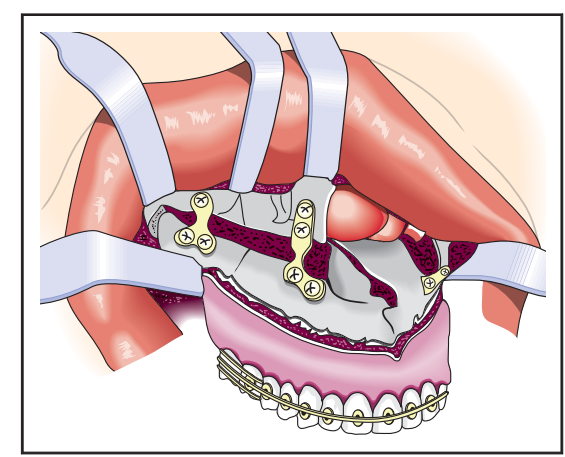

Abb. 33 Fertiggestellte Miniplattenosteosynthesen im typischen Muster mit Überbrückung der Pfeilerstrukturen - seitlich der Nasenapertur (parapiriform) und über der Cristae zygomaticoalveolaris.

syntheseplatten über den kaudalen Pfeilerstrukturen (parapiriform und Cristae zygomaticoalveolaris) [37] stabilisiert (Abb. 33). Nach Öffnen der intermaxillären Drahtligaturen muss sich der Unterkiefer zwanglos in den interokklusalen Splint führen lassen - ist dies nicht der Fall, so haben die Kiefergelenkköpfe während der Autorotation nicht korrekt in den Gelenkgruben gestanden.

Die Osteosynthesen müssen dann wieder gelöst und der Oberkiefer-Unterkiefer-Komplex erneut eingestellt werden.

Fall 3: Patientin, 42 Jahre, maxilläre Retrognathie (Abb. 34-36)

Block- bzw. Segmentosteotomien im Oberkiefer, Gaumennahterweiterung, Kortikalisschwächung

Zur Behandlung maxillärer Prognathie wurden Blockosteotomien bzw. Segmentosteotomien im Front-Eckzahn-Bereich angegeben. Bleiben die Osteotomielinien dabei unterhalb des Nasenbodens, 

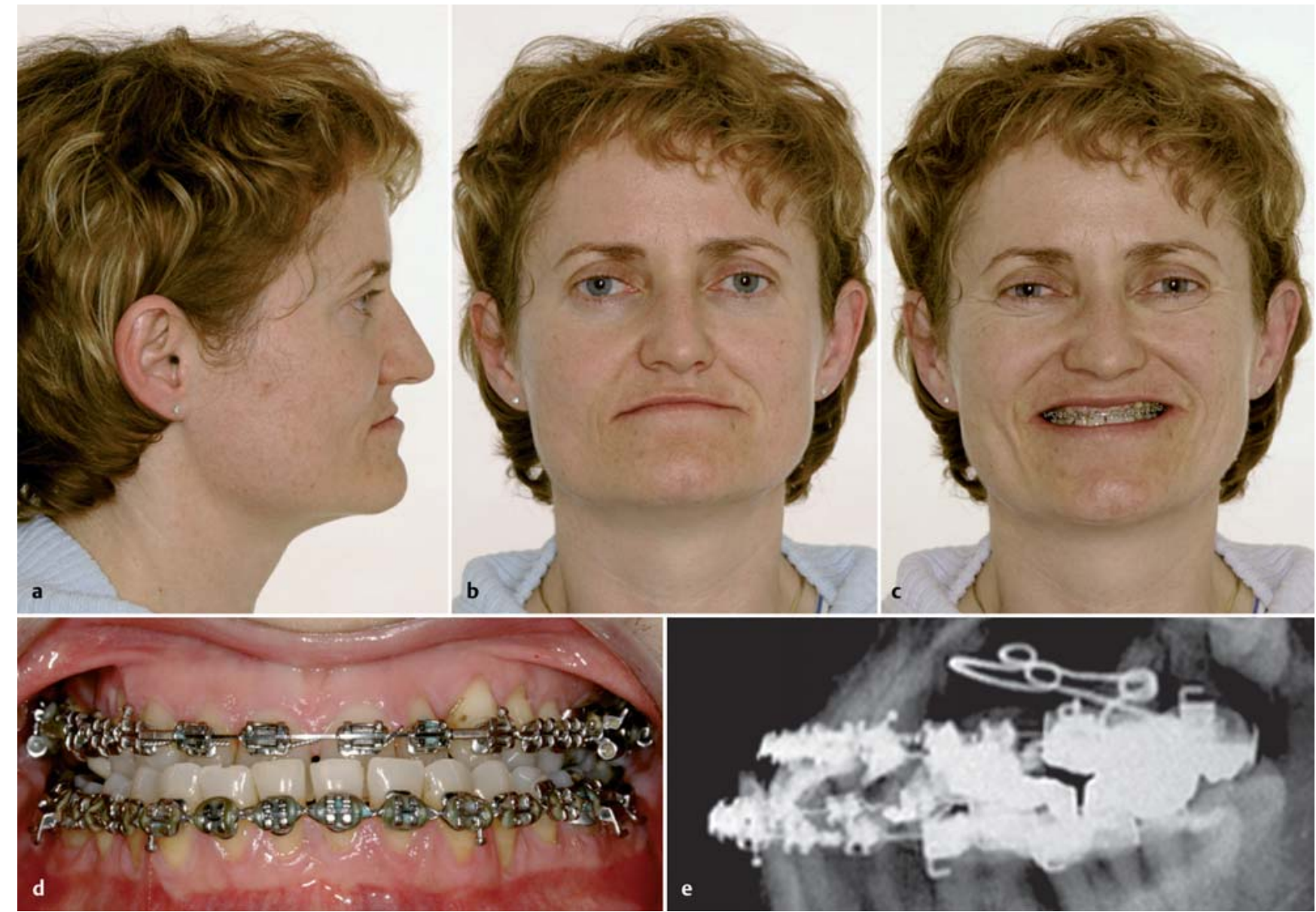

Abb. 34a bis e Präoperative Befunde Fall 3. a-c Konkaves seitliches Gesichtsprofil, flaches und breites Gesicht mit relativ großer Nase, flacher, eingefallener Oberlippe und positiver Lippentreppe als Ausdruck einer Rücklage des Oberkiefers. Das Gesicht wirkt vorgealtert. d Okklusale Beziehung nach kieferorthopädischer Vorbehandlung mit Multiband-Apparaturen - umgekehrter Überbiss. e Fernröntgenseitenbild mit Rücklage des Oberkiefers.

spricht man von einer Blockosteotomie, wird der Nasenboden durchtrennt, von einer Segmentosteotomie. Das Grundprinzip beider Varianten besteht in der Extraktion der vorderen Prämolaren, einer Ostektomie des knöchernen Zahnfachs, einer Querdurchtrennung ggf. mit Ektomie eines Knochenstreifens im Gaumendach und einer horizontalen Osteotomie unterhalb oder auf dem Niveau des Nasenbodens mit Abtrennung des Nasenseptums. Zur Aufrechterhaltung der Vaskularisation bei diesem Verfahren wurden verschiedene Schleimhautinzisionen als Zugangswege beschrieben: Bukkale Längsschnitte über den leeren Alveolen der Prämolaren, von denen aus die Schleimhaut nach median in Richtung auf den Nasenboden tunneliert und die Gaumenschleimhaut unterminiert wird (Trauner 1973) [17], die horizontale vestibuläre Schnittführung und Tunnelierung der übrigen Schleimhautpartien (Schuchardt/Köle) [38,39] sowie die alleinige palatinale Querinzision mit daraus resultierendem vestibulär-buk- kalem Schleinhautstiel (Wunderer 1962) [40].

Nach der Mobilisation kann das Knochenstück rückverlagert und/oder seine Inklination verändert werden. Die Osteosynthese mit Miniplatten nach Einstellung über interokklusale Splints kann sich je nach Zugangsweg schwierig gestalten.

Interessanterweise gibt es einen Vorläufer zu den heute im Rahmen von „accelerated“ oder "speedy orthognathics“ propagierten Kortikotomien: Um die kieferorthopädische Behandlung mit Spangen bei lückiger Protrusion in der Oberkieferfront zu erleichtern, wurden von Köle (1958) [41] bereits vertikale Kortikotomien in den verbreiterten Interradikularräumen empfohlen.

Die Oberkiefer-Seitenzahn-Segmentosteotomie nach Schuchardt [38] eignet sich zum Schluss eines frontal offenen Bisses mit korrekter vertikaler Position

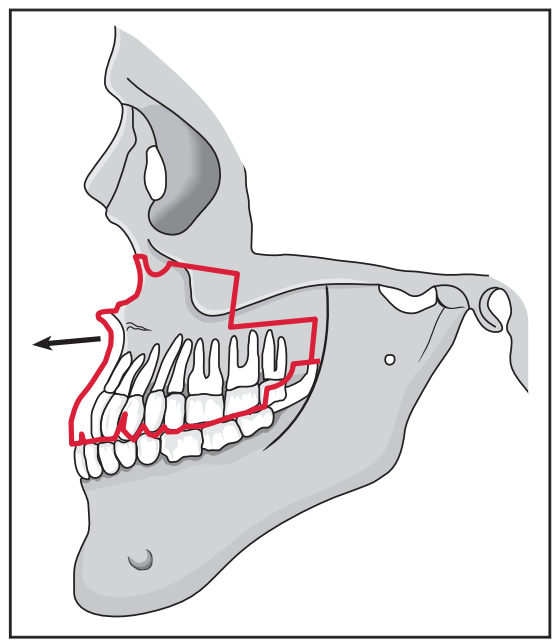

Abb. 35 Operationsschema Fall 3. Hohe LeFort-I-Osteotomie zur Oberkiefervorverlagerung.

der Oberkieferfrontzähne und normaler Lippenlänge, für den eine Elongation im Seitenzahngebiet verantwortlich ist. Die Seitenzahnsegmente werden von einem 


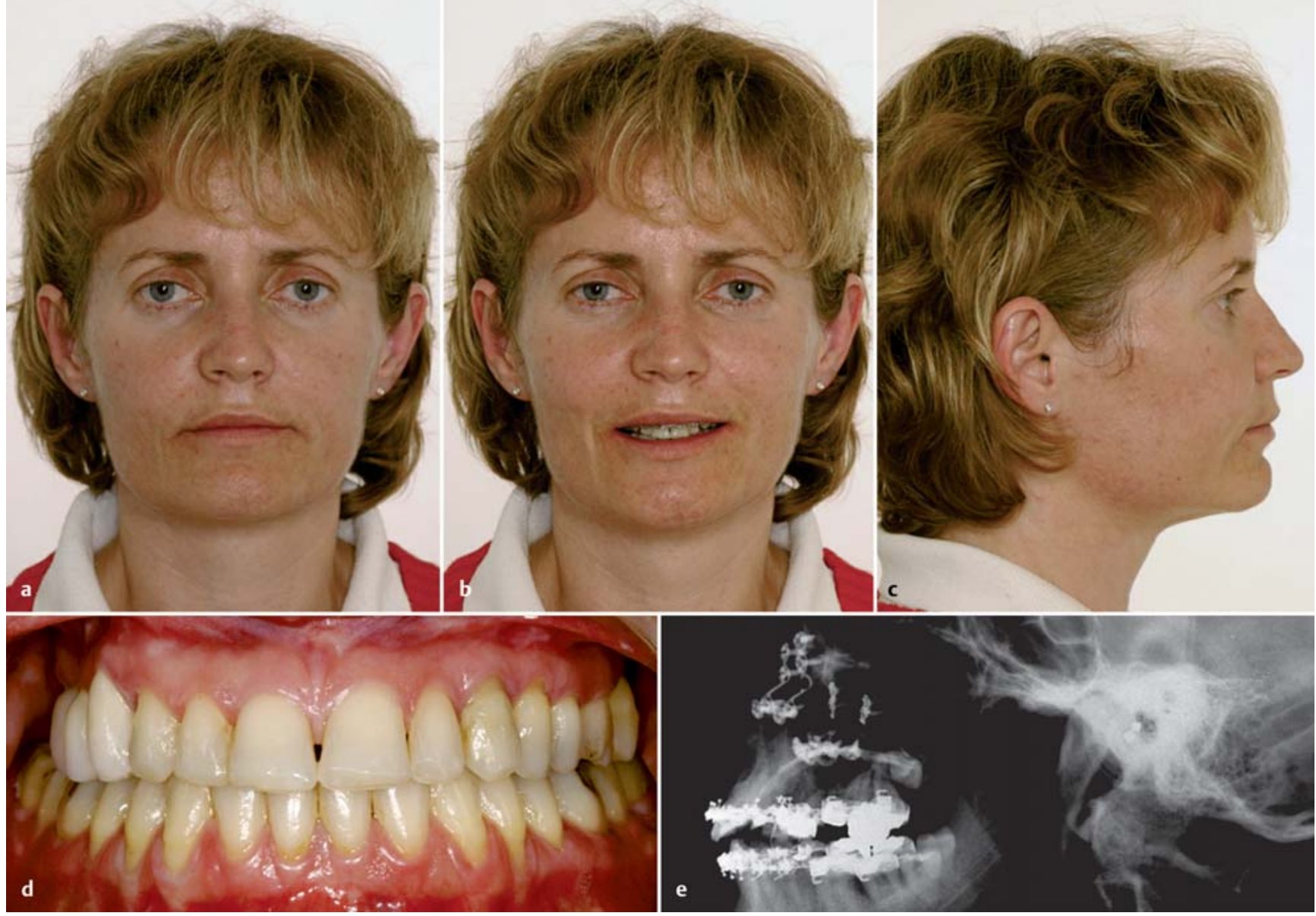

Abb. 36 a bis e Postoperative Befunde Fall 3. a-c Harmonisierung der Gesichtsproportionen mit Betonung des Mittelgesichts führt zu einem jüngeren Erscheinungsbild. d Okklusion in eugnather Verzahnung. e Fernröntgenseitenbild mit korrigierter Oberkieferposition und Osteosynthesematerial in situ.
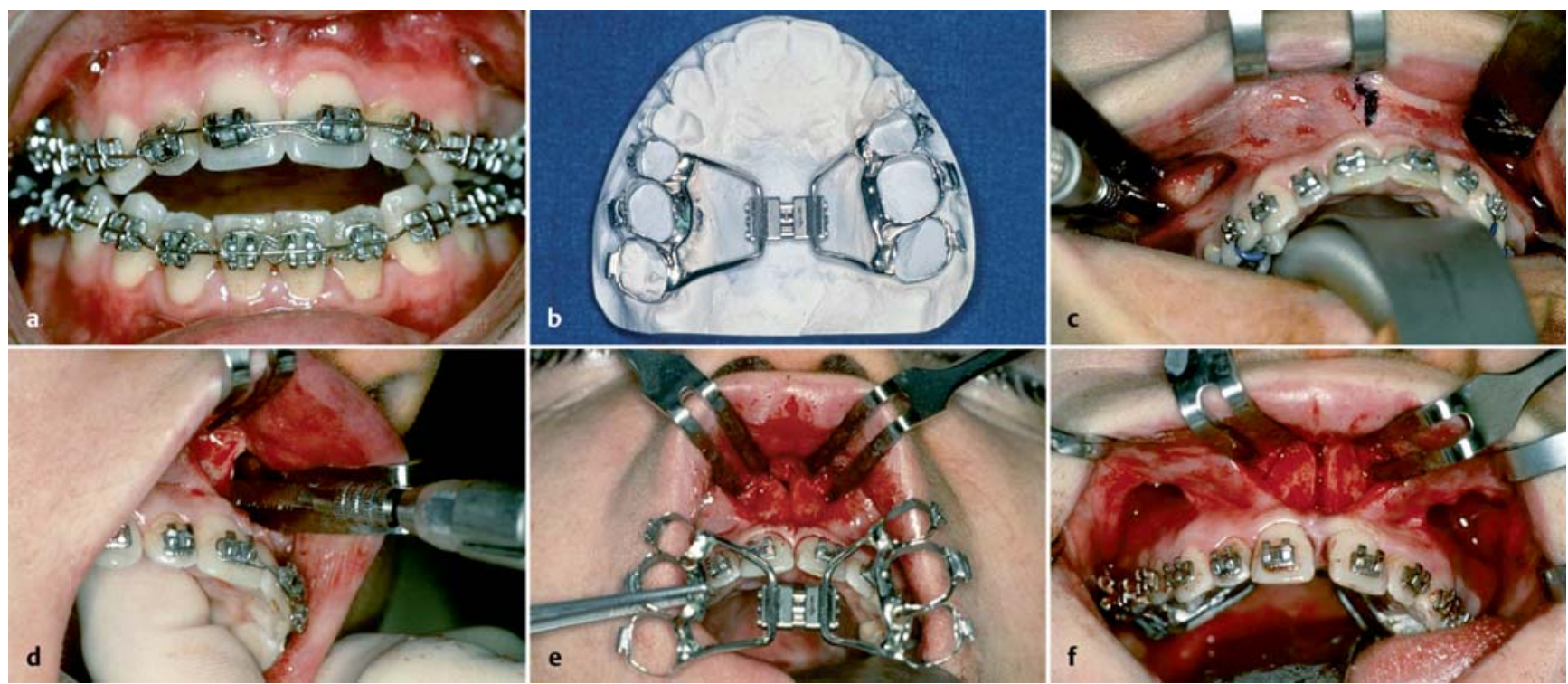

Abb. 37a bis f a Frontal offener Biss und transversaler Engstand im Oberkiefer mit Kreuzbisssituation im Seitenzahngebiet. b Zahngetragene Dehnschraube zu dem zugehörigen Oberkiefergipsmodell. c-f Osteotomien zur Schwächung der Cristae zygomaticoalveolares, median sagittale des Gaumens vom Nasenboden aus unter Palpationskontrolle, Einstzen der Dehnapparatur. Enstehung von Knochenspalten und eines Diastemas zwischen den mittleren Schneidezähnen nach Aktivierung der Dehnschraube. 

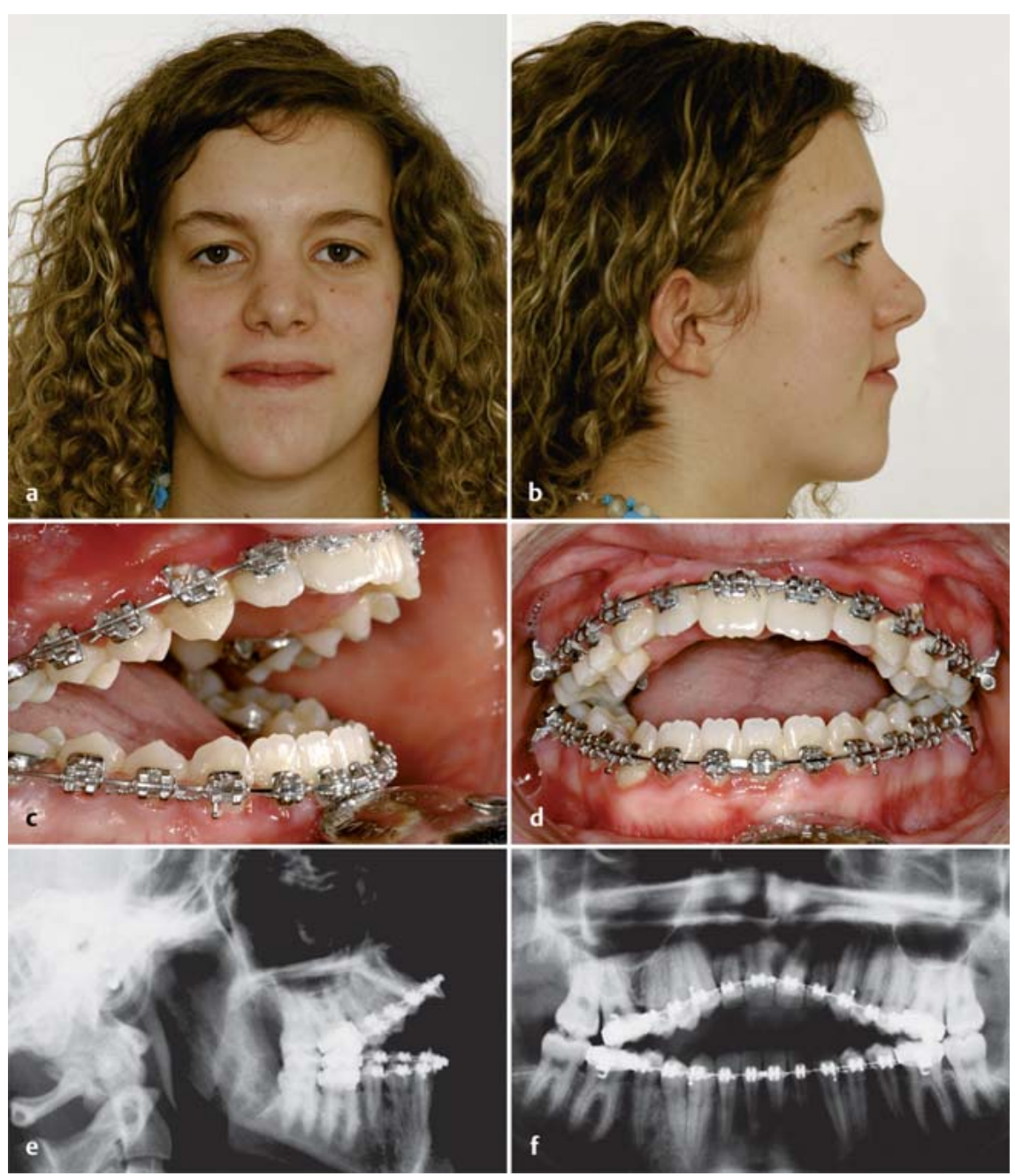

Abb. 38 a bis $\mathbf{f}$ Präoperative Befunde Fall 4. a, b Auffällige Verlängerung des unteren Gesichtsdrittels und Abweichung des Kinns nach rechts. c, d Okklusion mit deutlicher anteriorer Bissöffnung und isoliertem Zahnkontakt im Molarenbereich. e Fernröntgenseitbild zeigt die starke frontale Bissöffnung mit alveolärer und basal skeletaler Komponente. f Orthopantomogramm mit der präoperativen Situation nach Entfernung der Weisheitszähne.

vestibulären Zugang aus kastenförmig osteotomiert. Oberhalb der Wurzelspitzen bzw. im Bereich des Kieferhöhlenbodens wird bukkal ein Knochenstreifen ostektomiert und die palatinale Kortikalis über das entstandene Knochenfenster ausgefräst. Die Seitenzahnsegmente lassen sich dann mobilisieren, nach kranial verlagern und mit Miniplatten fixieren.

Zur transversalen Verbreiterung des Oberkiefers wird nach Ossifizierung der Sutur in der Mittellinie des Gaumens und extremem Engstand vielfach eine chirurgisch unterstützte Gaumennahterweiterung (GNE, Surgically assisted rapid palatinal expansion - SARPE) notwendig (Abb. 37). Das Design der Osteotomien hat kontinuierlich Änderungen und zunehmende Invasivität erfahren [42-45]:
Während in den Anfängen lediglich eine Osteotomie zur Schwächung der Mittelgesichtspfeiler (im Bereich der Cristae zygomaticoalveolares) mit einer medianen, meißelgeführten Durchtrennung der Gaumennaht und des Alveolarfortsatzes zwischen den mittleren Schneidezähnen unter digitaler Kontrolle auf der palatinalen Seite (Abb.34) kombiniert wurde, geht die Tendenz heute zur median sagittalen Teilung in Kombination mit einer kompletten Le-Fort-I-Osteotomie einschließlich pterygomaxillärer Sprengung, jedoch ohne Downfracture.

Nur mit einer Osteotomie in dieser Form lässt sich eine transversale Erweiterung mit Parallelstellung der Oberkiefersegmente in der gesamten Sagittalrichtung erzielen, wobei hohe Stressbelastungen im Bereich nicht osteotomierter Knochenbrücken vermieden werden [42].

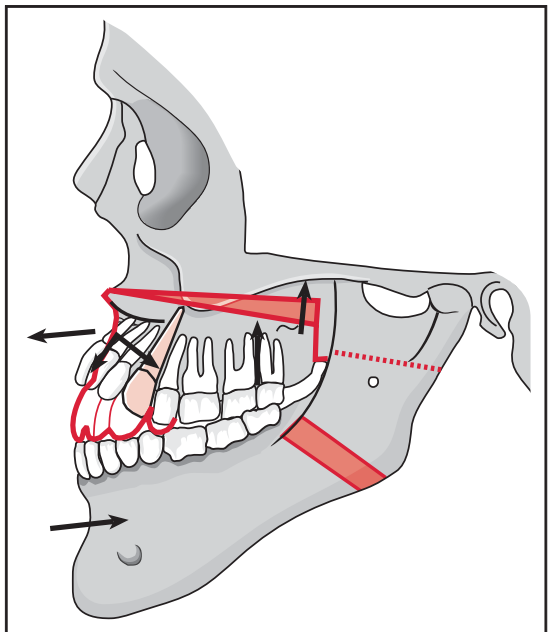

Abb. 39 Operationsschema Fall 4. Extraktion der vorderen Prämolaren (Zähne 14 und 24) im Oberkiefer. Le-Fort-I-Osteotomie und Segmentierung in 3 Teile (Anterior- und Seitenzahnsegmente). Dorsale Impaktierung, Abwärtsrotation des anterioren Segments. BSSO zur Unterkieferrückverlagerung.

Die Osteotomien werden über separate Schleimhautinzisionen im anterioren und lateralen Oberkiefer-Vestibulum vorgenommen, die durch Tunnel untereinander verbunden werden. Die median sagittale Osteotomie erfolgt vom unteren Nasengang aus (Abb.34). Als Modifikation dieser Technik wird über direkte Osteotomien der Gaumennaht nach palatinaler Schnittführung berichtet (Zoder) [45].

Die Gaumennaht wird mit zahngetragenen Dehnschrauben allmählich erweitert. Alternativ dazu sind auch spezielle knochengetragene Schraubenapparaturen verwendbar [11]. Die transversale Aufweitung des Oberkiefers erfolgt im Sinne einer Kallusdistraktion und geht mit Dehnung der Weichgewebe einher. Das Rezidivrisiko ist im Gegensatz zur 2-Piece-Le-Fort-I-Osteotomie mit akuter Aufweitung deshalb geringer.

In einigen Fällen reicht eine alleinige SARPE zur Korrektur einer skeletalen Dysgnathie aus - sehr viel häufiger ist sie aber der erste Schritt im Rahmen einer komplexeren Behandlung mit einer mono- oder bignathen Intervention im Anschluss.

Das meist verwendete Operationsverfahren zur Umstellung des Oberkiefers ist die Osteotomie auf der Le-Fort-I-Ebene mit Downfracture. Segmentosteotomien einzelner Zahngruppen spielen in heutiger Zeit eine untergeordnete Rolle. 


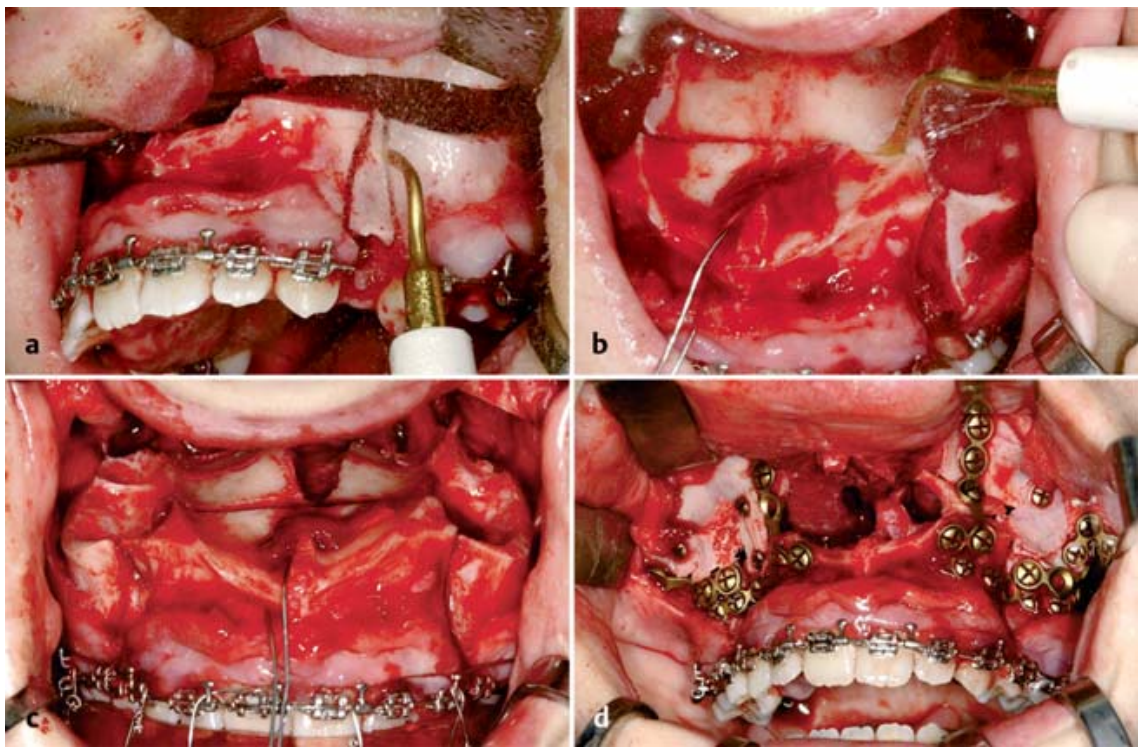

Abb. 40 a bis d Intraoperative Situation Fall 4. a Nach Extraktion der Zähne 14 und 24 und Osteotomie auf LeFort-I-Ebene wird mit dem piezochirurgischen Instrument ein Knochenkeil im Bereich der Zahnlücken ektomiert. b Nach Downfracture des Oberkiefers Blick auf den Nasenboden bzw. Hartgaumen von oben. Querdurchtrennung des Knochens auf dem Niveau der ektomierten Knochenkeile unter Erhalt der palatinalen Schleimhaut. Hierdurch entsteht ein isoliertes, gestieltes anteriores Knochensegment. c Nach median-sagittaler Osteotomie des hinteren Hartgaumens wird das frontale Segment unter Lückenschluss nach dorsal und abwärts bewegt und die Oberkieferbasis insgesamt dorsal angehoben (impaktiert). Einstellung aller Oberkieferblöcke mithilfe des interokklusalen Splints. d Fixierung der Oberkiefersegmente mit Osteosyntheseplatten. Füllung noch vorhandener Lücken mit Knochenstücken aus den Ostektomiebereichen. Die Zahnlücken 14 und 24 sind geschlossen.

\section{Bignathe Osteotomien}

Nicht aufgrund der Tatsache, dass sich Dysgnathien häufig in beiden Kiefern manifestieren, sondern auch zur Ergebnisoptimierung orthognath chirurgischer Operationen werden bignathe Korrekturen vorgenommen. Sämtliche hier angesprochenen Osteotomieverfahren können kombiniert werden. Die OP-Zeiten addieren sich bei einem bignathen Vorgehen. Im OP-Ablauf wird zur Positionierung des zuerst osteotomierten Kiefers ein interokklusaler Zwischensplint und zur Einstellung des 2. Kiefers ein definitiver Splint notwendig. Absolute Indikationen zum bignathen Vorgehen [1] ergeben sich bei:

- Kombination aus maxillärer Retrognathie und mandibulärer Prognathie mit großer sagittaler Stufe

- Gesichtsskoliose mit einseitiger vertikaler Verkürzung von Ober- und Unterkiefer

- Kombinationen von mandibulärer Laterognathie mit maxillärer Retrognathie.

Fall 4: Patientin, 18 Jahre, ausgeprägter frontal offener Biss, mandibuläre Laterognathie (Abb. 38-41)
Fall 5: Patientin, 24 Jahre, hemimandibuläre Hyperplasie links (Abb. 42-44)

\section{Verfahrensabhängige Komplikationen}

Bei allen orthognathen chirurgischen Operationsverfahren besteht ein Risiko für Komplikationen. Intraoperative Komplikationen wie irreguläre Frakturen, Nervenläsionen und massive Blutungen sind typisch.

Während der sagittalen Spaltungsosteotomie im aufsteigenden Unterkieferast kommt es gelegentlich zu einem sog. „bad split“. Durch Absprengungen und Fragmentierung kann die Kontinuität der Außen- und/oder Innenkortikalis zum Processus condylaris bzw. mit der Unterkieferspange verlorengehen (Abb.45). Ein ,bad split“ kann sich als Folge abnormer anatomischer Verhältnisse mit extrem graziler Dimensionierung des aufsteigenden Astes ergeben. Weitere Gründe sind inadäquate Technik und/oder der Einsatz ungeeigneter Instrumente. Eine osteosynthetische Versorgung der atypischen Fraktur nach den traumatologischen Gesichtspunkten ist obligat, Schwierigkeiten resultieren dabei aus der „heterotopen“ Fragmentstellung in-

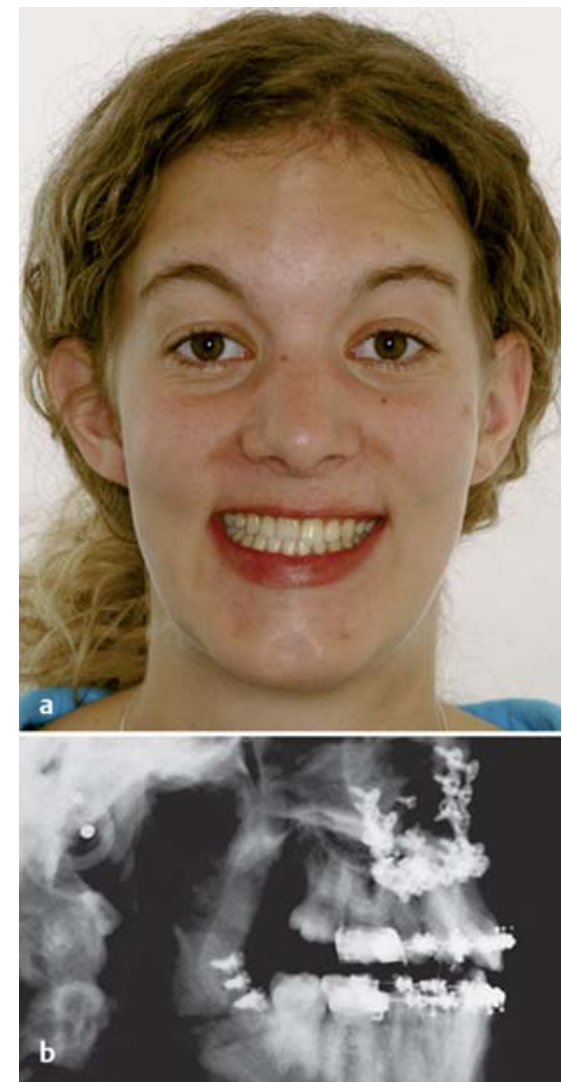

Abb. 41 a und b Postoperative Befunde Fall 4.

folge der Umstellungsosteotomie. In seltenen Fällen bedarf es eines externen Zugangs mit Inzision der Gesichtshaut. Postoperativ muss entschieden werden, ob eine zusätzliche intermaxilläre Ruhigstellung des Unterkiefers die knöcherne Konsolidierung günstig beeinflussen kann.

Bei Osteotomien im Unterkieferkörper dorsal des Foramen mentale und im $\mathrm{R}$. ascendens ist mit Eröffnungen des Mandibularkanals und Verletzungen des $\mathrm{N}$. alveolaris inferior zu rechnen. Die intraoperativen Nervenläsionen können durch Druck, Zug und aus Kontinuitätsverlusten resultieren [46-49].

Nach Osteotomien im Unterkiefer wurden von 40-70\% der Patienten Gefühlsstörungen im Bereich der Unterlippe und der bukkalen Gingiva angegeben [49], die 1 Jahr postoperativ noch in 1$10 \%$ der Fälle persistierten [46-50].

In Schnittbildern aus der präoperativen Bildgebung (DVT, CT) kann der Verlauf des N. alveolaris inferior dargestellt werden, um den Mandibularkanal während der Osteotomie zu umgehen und das Risiko einer Nervenläsion zu senken. In 

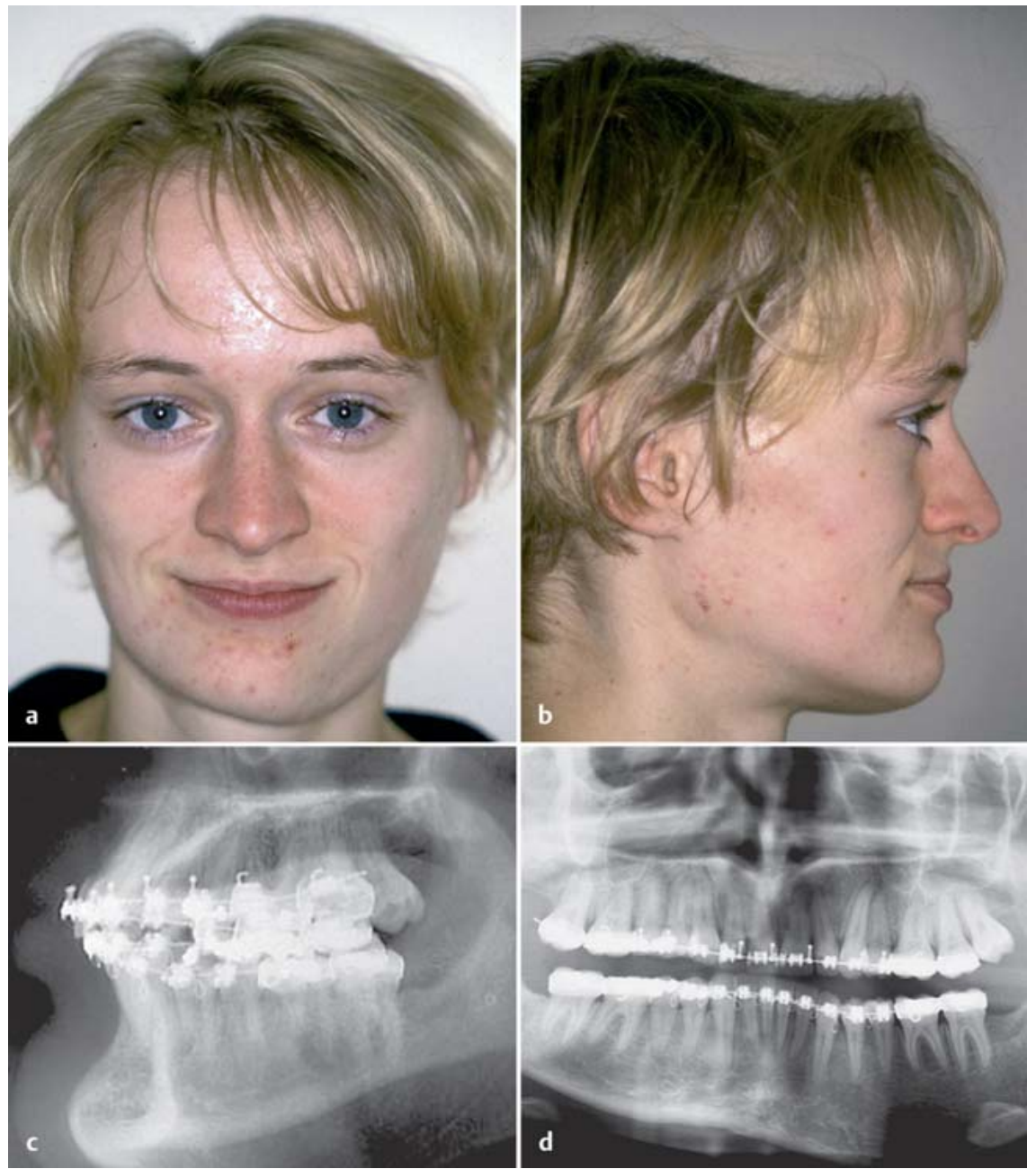

Abb. 42 a bis d Präoperative Befunde Fall 5. a, b Asymmetrie des Untergesichts mit tiefstehendem Unterkieferrand links. c, d Fernröntgenseitenbild und Orthopantomogramm. Hemimandibuläre Hyperplasie links und Tendenz zur Hypoplasie der Unterkieferhälfte rechts. Kompensation der Kaudalposition im Unterkiefer links durch Supraokklusion im Oberkieferseitenzahnbereich und Transversalneigung („Canting“) der Okklusionsebene.

Einzelfällen wurden bei Osteotomien im Ramusbereich auch Läsionen des N. fazialis beobachtet. Der Stamm dieser Nerven liegt in direkter topografischer Beziehung zum Hinterrand des aufsteigenden Astes.

Im Rahmen von Oberkieferosteotomien sind Komplikationen viel seltener als im Unterkiefer. Sensibilitätsstörungen im Bereich der vestibulären und palatinalen Schleimhaut sowie der Oberlippe und im Wangenbereich treten nach Durchtrennung des $\mathrm{N}$. inzisivus und Läsionen des $\mathrm{N}$. infraorbitalis auf. Sie sind in der Regel temporär und vollständig reversibel. Bei Oberkieferosteotomien, die bis nach retrotubär gehen, können Verletzungen größerer Gefäße, wie der A. maxillaris und sphenopalatina oder des Plexus pterygoideus, auftreten. Sie erfordern gezielte intraoperative Blutstillungsmaß- nahmen, unter Umständen zwingen sie auch zum OP-Abbruch und zur Katheterangiografie und Embolisation.

Als äußerst rare, aber besonders schwerwiegende Komplikation sind Erblindungen nach Le-Fort-I-Osteotomien beschrieben [51,52]. Wahrscheinlich sind sie Folge irregulärer Frakturen, die über das Gaumen- und Keilbein bis in den Optikuskanal vordringen und auf eine forcierte Downfracture zurückführbar sind.

Postoperativ können Durchblutungsstörungen im Bereich osteotomierter Knochenabschnitte auftreten, die sich in Nekrosen von Schleimhaut und Knochen mit Verlust von Zähnen oder ganzen Kieferanteilen äußern. Die Gefahr von Beeinträchtigungen der Vaskularisation nimmt zu, wenn kleine Blöcke oder Segmente gebildet werden.



Abb. 43 a und $\mathbf{b}$ Operationsschema Fall 5. Bignathe Operation. Asymmetrische Le-Fort-IOsteotomie zur Vorverlagerung des Oberkiefers, gleichzeitig mit dorsaler Impaktierung und transversaler Derotation. BSSO zur Rückverlagerung des Unterkiefers, ebenfalls mit transversaler Derotation, zur Einstellung der Okklusionsebene im Rahmen der sagittalen Spaltung links, Lateralisation des N. alveolaris inferior links, um den Basalrand des Unterkiefers abzutragen. Augmentation des Unterkieferunterrands rechts mit dem links entnommenen Knochenmaterial.

Die Gründe für postoperative Instabilitäten oder bindegewebige Pseudoarthrosen (Non-Union) sind außer in Durchblutungsstörungen in mangelndem intersegmentalem Knochenkontakt bzw. übergroßen Knochenlücken und/ oder inadäquaten Osteosynthesen [53] zu suchen. Abhilfe können nur die Reoperation, Überbrückung mit Knochentransplantaten und eine geeignete Fixierungstechnik schaffen [37].

Reoperationen mit Neupositionierung der Segmente in Unter- oder Oberkiefer werden auch notwendig, wenn sich 


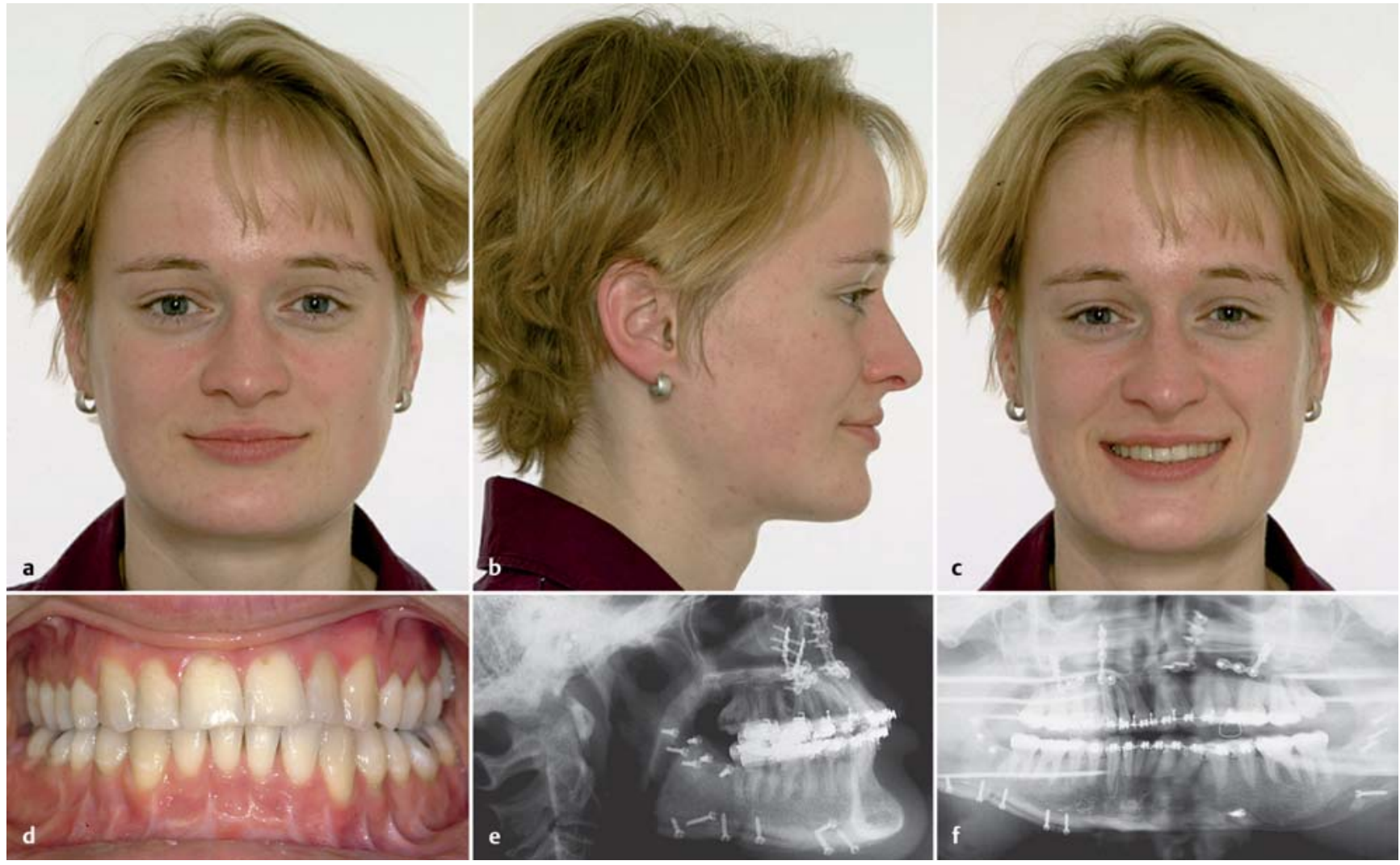

Abb. 44 a bis $\mathbf{f}$ Postoperative Befunde Fall 5 nach 1 Jahr. a-d Weitgehende Herstellung der Gesichtssymmetrie im Bereich der Kieferbasen und der Okklusionsebene. e, $\mathbf{f}$ Fernröntgenseitenbild und Orthopantomogramm. Osteosynthesematerial nach Oberkiefervor- und Unterkieferrückverlagerung. Die links basal am Unterkieferrand abgetragenen und zur Augmentation nach rechts transplantierten Knochenspäne sind mit insgesamt 5 Schrauben fixiert.



Abb. 45 Orthopantomogramm nach BSSO mit „bad split“ links. Eine irregulär abgesprengte Knochenlamelle von der Vorderkante der Außenkortikalis wurde durch transorale Applikation zusätzlicher Miniplatten refixiert.

postoperativ durch orthodontische Nachbehandlung nicht ausgleichbare Malokklusionen, Zwangsbisse oder Fehlstellungen der Kiefergelenke zeigen. Von der Weiterentwicklung atraumatischer Osteotomietechniken mit Unterstützung durch Endoskopie [54] und Robotik wird eine Verbesserung der Präzision, Effizienz und Sicherheit orthognath chirurgischer Eingriffe erwartet.

\section{Zusammenfassung}

Die derzeit üblichen Operationsverfahren und Osteotomietechniken der orthognathen Chirurgie sind etablierte Verfahren, deren Risiko kalkulierbar ist.

\section{Literatur}

${ }^{1}$ Mast G, Ehrenfeld M. Chirurgische Kieferorthopädie. In: Schwenzer N, Ehrenfeld M, Hrsg. Zahn-Mund-Kiefer-Heilkunde, MundKiefer- und Gesichtschirurgie. 4. Aufl. Stuttgart: Thieme; 2011: 233-261

2 Steinhäuser E, Janson I. Kieferorthopädische Chirurgie. Band I, Eine interdisziplinäre Aufgabe. Berlin: Quintessenz; 1988

${ }^{3}$ Arnett GW, Gunson MJ. Esthetic treatment planning for orthognathic surgery. J Clin Orthod 2010: 3: 196-200

${ }^{4}$ Krenkel C, Lixl G. Model surgical apparatus for planning and simulation of maxillary and mandibular osteotomies. Zahnärztl Prax 1991; 42: 471-473

${ }^{5}$ Bell WH, Fenn RA, Buschang PH. Accelarated orthognathic surgery and increased orthodontic efficiency: a paradigm shift. J Oral Maxillofac Surg 2009; 67: 2043-2044

${ }^{6}$ Bell WH, Finn RA. Accelerated orthognathic surgery and increased orthodontic efficiency: a paradigm shift. J Oral Maxillofac Surg 2011; 69: 579-583

7 Wilcko MT, Wilcko WM, Pulver JJ et al. Accelerated osteogenic orthodontics: a 1 stage surgically facilitated rapid orthodontic technique with alveolar augmentation. J Oral Maxillofac Surg 2009; 67: 2149-2159

8 Swennen GR, Mollemans W, Schutyser F. Threedimensional treatment planning of orthognathic surgery in the era of virtual imaging. J Oral Maxillofac Surg 2009; 67: 2080-2092

9 Swennen GR, Mollemans W, De Clercq Cet al. A cone-beam computed tomography triple scan procedure to obtain a three-dimensional augmented virtual skull model appropriate 
for orthognathic surgery planning. J Craniofac Surg 2009; 20: 297-307

${ }^{10}$ Swennen GRJ, Schutyser F, Hausamen JE. Three dimensional Cephalometry, a Color Atlas and Manual. Berlin, Heidelberg, New York: Springer; 2006

11 Westermark A, Zachow S, Eppley B. 3-D osteotomy planning in maxillofacial surgery, including soft tissue prediction. J Craniofac Surg 2005; 16: 100-104

12 Schramm A, Gellrich NC, Schmelzeisen R. Navigational Surgery of the facial Skeleton. Berlin, Heidelberg, New York: Springer; 2007

${ }^{13}$ Metzger MC, Hohlweg-Majert B, Schwarz U et al. Manufacturing splints for orthognathic surgery using a three-dimensional printer Oral Surg Oral Med Oral Pathol Oral Radiol Endod 2008; 105: e1-e7

14 Triaca A, Minoretti R, Saulacic N. Mandibula wing osteotomy for correction of the mandibular plane: a case report. Br J Oral Maxillofac Surg 2010; 48: 182-184

15 Trauner R, Obwegeser H. The surgical correction of mandibular prognathism and retrognathia with consideration of genioplasty. I. Surgical procedures to correct Mandibular prognathism and reshaping of the chin. Oral Surg Oral Med Oral Pathol 1957; 10: 677-689

${ }^{16}$ Trauner R, Obwegeser $H$. The surgical correction of mandibular prognathism and retrognathia with consideration of genioplasty. II Operating methods for microgenia and distoclusion. Oral Surg Oral Med Oral Pathol 1957; 10: 787-792

17 Trauner R. Kiefer- und Gesichtschirurgie. München, Berlin, Wien: Urban und Schwarzenberg; 1973

18 Robiony M, Polini F, Costa F et al. Piezoelectric bone cutting in multipiece maxillary osteotomies. J Oral Maxillofac Surg 2004; 62: 759761

${ }^{19}$ Hunsuck EE. A modified intraoral sagittal splitting technic for correction of mandibular prognathism. J Oral Surg 1968; 26: 250-253

20 Dal Pont G. Retromolar osteotomy for the correction of prognathism. J Oral Surg Anesth Hosp Dent Serv 1961; 19: 42-47

${ }^{21}$ Epker BN. Modifications in the sagittal osteotomy of the mandible. J Oral Surg 1977; 35 157-159

22 Perthes G. Über Frakturen und Luxationsfrakturen des Kieferköpfchens und ihre operative Behandlung. Langenbecks Arch klein Chir 1924; 133: 418-429

23 Schuchardt K. Die Chirurgie als Helferin der Kieferorthopädie. Fortschr Kieferorthop 1954; 15: 11-13

24 Zizelmann C, Hammer B, Gellrich NC et al. In vitro biomechanical comparison of the effect of pattern, inclination, and size of positional screws on load resistance for bilateral sagittal split osteotomy. J Oral Maxillofac Surg 2011; 69: 1458-1463

25 Borstlap WA, Stoelinga PJ. Hoppenreijs TJ et al. Stabilisation of sagittal split advancement osteotomies with miniplates: a prospective multicentre study with two-year follow-up. Part I. Clinical parameters. Int J Oral Maxillofac Surg 2004; 33: 433-441

${ }^{26}$ Borstlap WA, Stoelinga PJ, Hoppenreijs TJ et al. Stabilisation of sagittal split advancement osteotomies with miniplates: a prospective, multicentre study with two-year follow-up. Part II. Radiographic parameters. Int J Oral Maxillofac Surg 2004; 33: 535-542

27 Maurer P, Knoll WD, Schubert J. Comparative evaluation of two osteosynthesis methods on stability following sagittal split ramus osteotomy. J Craniomaxillofac Surg 2003; 31: 284-289
${ }^{28}$ Kleier C, Kleinheinz J, Stamm T et al. Prospektive kephalometrische Evaluation eines neuen dreidimensional justierbaren Osteosynthesesystems fur die sagittale Ramusosteotomie. Mund Kiefer Gesichtschir 2000; 4: 296-300

${ }^{29}$ Hofer O. Die operative Behandlung der alveolären Retraktion des Unterkiefers und ihre Anwendungsmöglichkeit für Prognathie und Mikrogenie. Dtsch Zahn Mund Kieferheilk 1942; 9: 142-144

${ }^{30}$ Dingman RO. Surgical correction of mandibular prognathism, an improved method. Am J Orthodont Oral Surg 1944; 11: 683-692

31 Converse JM, Shapiro HH. Bonegrafting for malformations of the jaws: cephalometric diagnosis in the surgical treatment of malformations of the face. Am J Surg 1954; 88 : 858-863

32 Cheever DW. Displacement of the upper jaw. First Med. Surg. Rep. Boston City Hosp. 156, 1870. Zitiert nach Halvorson E, Mulliken JB. Cheever's double operation: the first Le Fort I osteotomy. Plast Reconstr Surg 2008, 121: 1375-1381

${ }^{33}$ Wassmund M. Lehrbuch der praktischen Chirurgie des Mundes und der Kiefer. Band I. Leipzig: Johann Ambrosius Barth Verlag; 1935 (Beschreibung der Wassmund-Fraktur bereits 1927)

${ }^{34}$ Axhausen G. Zur Behandlung veralterter dislozierter Oberkieferbrüche. Dtsch Zahn Mund Kieferheilk 1934; 1: 334-338

35 Bell WH, Fonsec RJ, Kennedy JW et al. Bone healing and revascularization after total maxillary ostetomy. J Oral Surg 1975; 33 : 253-260

${ }^{36}$ Epker BN. Vascular considerations in orthognathic surgery. II. Maxillary osteotomies. Oral Med Oral Surg Oral Pathol 1984; 57: 473-478

37 Klammert U, Müller-Richter UDA, Böhm $\mathrm{H}$ et al. Aktuelle Osteosyntheseverfahren in der orthognathen Chirurgie. MKG-Chirurg 2011; 4: $171-179$

38 Schuchardt K. Formen des offenen Bisses und ihre operativen Behandlungsmöglichkeiten. Fortschr Kiefer Gesichtschir 1955; 1: 222

${ }^{39}$ Köle $H$. Formen des offenen Bisses und ihre chirurgische Behandlung. Dtsch Stomatol 1959: 9: 753-757

${ }^{40}$ Wunderer S. Die Prognathieoperation mit frontal gestieltem Maxillafragment. Öst Z Stomatol 1962; 59: 98-101

${ }^{41}$ Köle H. Corticalisschwächung zur Unterstützung bei der kieferorthopädischen Behandlung. Fortschr Kiefer Gesichtschir 1958; 4: 161-166

42 Holberg C, Steinhäuser S, Rudzki-Janson I. Rapid maxillary expansion in adults:cranial stress reduction depending on the extent of surgery. Eur J Orthod 2007; 29: 31-36

${ }^{43}$ Mommaerts MY. Transpalatinal distraction as a method of maxillary expansion. Br J Oral Maxillofac Surg 1999; 37: 268-272

${ }^{44}$ Krey KF, Walter F, Dannhauer KH. Changes in arch form following surgically-assisted rapid maxillary expansion (SRME). J Orofac Orthop 2008: 69: 177-188

${ }^{45}$ Zoder W, Eisenhart G, Wangerin K. Therapie transversaler Diskrepanzen in der orthognathen Chirurgie. MKG-Chirurg 2011; 4: 160170

46 Westermark A, Bystedt $H$, von Konow L Inferior alveolar nerve function after sagittal split osteotomy of the mandible: correlation with degree of intraoperative nerve encounter and other variables in 496 operations. $\mathrm{Br} \mathrm{J}$ Oral Maxillofac Surg 1998; 36: 429-433

47 Westermark A, Bystedt $H$, von Konow L. Inferior alveolar nerve function after mandibular osteotomies. Br J Oral Maxillofac Surg 1998; 36: 425-428

${ }^{48}$ Westermark A, Englesson L, Bongenhielm U. Neurosensory function after sagittal split osteotomy of the mandible: a comparison between subjective evaluation and objective assessment. Int J Adult Orthodon Orthognath Surg 1999; 14: 268-275

${ }^{49}$ Teerijoki-Oksa T, Jaaskelainen SK, Forssell K. Risk factors of nerve injury during mandibular sagittal split osteotomy. Int J Oral Maxillofac Surg 2002; 31: 33-39

50 Nesari S, Kahnberg KE, Rasmusson L. Neurosensory function of the inferior alveolar nerve after bilateral sagittal ramus osteotomy: a retrospective study of 68 patients. Int J Oral Maxillofac Surg 2005; 34: 495-498

${ }^{51}$ Lanigan DT, Romanchuk K, Olson CK. Ophthalmic complications associated with orthognathic surgery. J Oral Maxillofac Surg 1993; 51: 480-494

52 Steel BJ, Cope MR. Unusual and rare complications of orthognathic surgery: a literature review. J Oral Maxillofac Surg 2011; Epub ahead of print

53 Ow A, Cheung LK. Skeletal stability and complications of bilateral sagittal split osteotomies and mandibular distraction osteogenesis: an evidence based review. J Oral Maxillofac Surg 2009; 67: 2344-2353

${ }^{54}$ Mommaerts MY. Endoscopically assisted sagittal split osteotomy for mandibular lengthening: technical note and initial experience. J Craniomaxillofac Surg 2010; 38: 108-112

Dr. med. Dr. med. dent. Martin Roser Leitender Oberarzt

Klinik für Mund-, Kiefer- und

Gesichtschirurgie

Klinikum Konstanz

Luisenstraße 7

78464 Konstanz

martin.roser@klinikum-konstanz.de

Prof. Dr. med. Dr. med. dent.

Gerd Gehrke

Ärztlicher Direktor

Klinik für Mund-, Kiefer- und

Gesichtschirurgie

Plastische Operationen

Henriettenstiftung

Marienstraße 72-90

30171 Hannover

Prof. Dr. med. Dr. med. dent.

Carl Peter Cornelius

Oberarzt

Prof. Dr. med. Dr. med. dent.

Michael Ehrenfeld

Ärztlicher Direktor

Dr. med. Dr. med. dent. Gerson Mast

Leitender Oberarzt

Klinik und Poliklinik für Mund-,

Kiefer- und Gesichtschirurgie

LMU München

Lindwurmstraße $2 \mathrm{a}$

80337 München 